\title{
Chapter 15 \\ Conceptual Modeling Foundations for a Web of Knowledge
}

\author{
David W. Embley and Stephen W. Liddle and Deryle W. Lonsdale
}

\begin{abstract}
The semantic web purports to be a web of knowledge that can answer our questions, help us reason about everyday problems as well as scientific endeavors, and service many of our wants and needs. Researchers and others expound various views about exactly what this means. Here we propose an answer with conceptual modeling as its foundation. We define a web of knowledge as a collection of interconnected knowledge bundles superimposed over a web of documents. Knowledge bundles are conceptual model instances augmented with facilities that provide for both extensional and intensional facts, for linking between knowledge bundles yielding a web of data, and for linking to an underlying document collection providing a means of authentication. We formally define both the component parts of these augmented conceptual models and their synergistic interconnections. As for practicalities, we discuss problems regarding the potentially high cost of constructing a web of knowledge and explain how they may be mitigated. We also discuss usage issues and show how untrained users can interact with and gain benefit from a web of knowledge.
\end{abstract}

\subsection{Introduction}

Ideas about the semantic web have been with us ever since Tim BernersLee's published his book, Weaving the Web [BL99], and his Scientific American article, The Semantic Web [BLHL01], with Hendler and Lassila. They and others have continued to discuss these ideas in an effort to more fully explain the semantic-web vision - its practicalities, successes, and challenges

David W. Embley, Stephen W. Liddle, Deryle W. Lonsdale

Brigham Young University, Provo, Utah 84602, USA, e-mail: embley@cs.byu.edu, liddle@byu.edu, lonz@byu.edu 
[SHBL06, AH08]. The W3C web site introduces the semantic web simply as "a web of data" [W3C].

Many of these ideas hark back even to the days of Plato $[\mathrm{PlaBC}]$ and Aristotle $[\mathrm{AriBC}]$ and the beginnings of philosophical discussions about ontology, epistemology, and logic. Here, we begin with this ancient view of semantics and show how it leads to a view of the semantic web rooted in conceptual modeling. In particular, we show how conceptual modeling can unify a view of these fundamental concepts and provide a practical way to realize them. Our intent is not to resolve questions about what the semantic web is, but rather to provide a practical view of one possible path toward realizing some of the benefits claimed by semantic-web visionaries. We call our conceptualmodeling view of the semantic web a "Web of Knowledge" (a "WoK").

To motivate our vision of a WoK, consider the current web of pages, which contains a wealth of knowledge. Unfortunately, most of the knowledge is not encoded in a way that enables direct user query. We cannot, for example, directly google for a car that is a 2003 or newer selling for under 15 grand; or for the names of the parents of great-grandpa Schnitker; or for countries whose population will likely decrease by more than $10 \%$ in 50 years. A way to enable direct query for facts embedded in web pages and facts implied by these stated facts is to annotate facts with respect to ontologies. Annotating facts implicitly populates these ontologies, turning them into a database over which structured queries can be executed. Annotation links also provide a form of provenance and authentication, allowing users to verify query results by checking original sources. Furthermore, facts and ontological concepts may appear in more than one populated ontology. Linking facts and ontological concepts across ontologies can provide navigation paths to explore additional, related knowledge. The web with a superimposed layer of interlinked ontologies each annotating a myriad of facts from the underlying web becomes a Web of Knowledge, a WoK.

Although this vision of a WoK is appealing, there are significant barriers preventing both its creation and its use. Ontology languages exist, with OWL being the de facto standard. RDF files can provide data for these ontologies and can also store annotation information linking data to facts in web pages and linking equivalent information in RDF files to one another. The SPARQL query language is a standard for querying RDF data. SWRL rules can provide for reasoning. Thus, all constituent components for a WoK are W3C standards in common use, and they even all work together allowing for immediate WoK development and usage. Nevertheless, the barriers of creation and usage remain high and effectively prevent WoK deployment. The creation barrier is high because of the cost involved in developing OWL ontologies and annotating web pages by linking RDF-encoded facts in web pages to these OWL ontologies. The usage barrier is high because untrained users cannot write SPARQL queries and SWRL rules.

In this exposition, we show how conceptual modeling can enable a WoKcan provide a firm foundation for a WoK and ways to break through the 
barriers to WoK creation and usage. We begin in Section 15.2 by discussing a computational view of ontology, epistemology, and logic. We argue that conceptual models, augmented in a particular way, build nicely upon these philosophical ideas so as to enable a WoK. In Section 15.3 we formalize this foundation. The formalization leads to a clear understanding of what must be done to create a WoK and make it usable. We then discuss initiatives we have investigated to address these challenges and opportunities - for construction in Section 15.4 and for usage in Section 15.5. We conclude in Section 15.6.

\subsection{WoK Conceptualization}

To think about constructing and using a web of knowledge, we first ask some fundamental questions: What is data? What are facts? What is knowledge? How does one reason and know? Philosophers have pursued answers to these questions for millennia; and although we do not pretend to be able to contribute to philosophy, we can use their ideas about ontology, epistemology, and logic to guide us in how to build and use a WoK.

- Ontology is the study of existence. It asks: "What exists?" In our quest to build a WoK, we must find computational solutions the question: "What concepts, relationships, and constraints exist?" We answer computationally, saying that we can declare a formal conceptual model for some domain of knowledge that captures the relevant concepts along with the relationships among these concepts and the constraints over these concepts and relationships. ${ }^{1}$

- Epistemology is the study of the nature of knowledge. It asks: "What is knowledge?" and "How is knowledge acquired?" To build a WoK, we provide computational answers to "What is digitally stored knowledge?" and "How does raw data become algorithmically accessible knowledge?" Our answer is to turn raw data into knowledge by populating conceptual models - by embedding facts in the concepts and relationships in accord with constraints. We further follow Plato's lead in wanting our knowledge to be justified $[\mathrm{PlaBC}]$, and thus we provide (1) annotation links that connect facts embedded in ontologies to sources from which they are extracted and (2) data and concept "same-as" connections that link objects and concepts across populated ontologies.

- Logic comprises principles and criteria of valid inference. It asks: "What is known?" and "What can be inferred?" In the computational context

\footnotetext{
${ }^{1}$ Purists argue that conceptual models are not ontologies [Gru93, Gua98, Smi03]. We agree that when conceptual models play their traditional role to aid in database schema design, they typically are not ontologies. But when they are used to answer "What exists?" and thus when they formally capture the concepts, relationships, and constraints that exist in a domain, they are ontologies.
} 
of a WoK, it can answer the question: "What are the known facts, both given and implied?" We ground our conceptual model in a description logic - a decidable fragment of first-order logic [BN03]. To make this logic practical for non-logicians, we must and do add a query generator whose input consists of ordinary free-form textual expressions or ordinary fillin-the-blank query forms. Both query modes fundamentally depend on conceptual-model-based ontologies to convert free-form and form-based queries to structured queries. Justification of query results relies on tracing annotation links back to source data and on following reasoning chains.

To illustrate these ideas, we give some examples. Suppose we wish to find a used car to purchase. We might pose this query: "Find me a red Nissan for under $\$ 5000$, a 1990 or newer with less than $100 \mathrm{~K}$ miles on it", or this query: "I'd like a Japanese-made car for under 15 grand." Figure 15.1 shows some web pages with cars for sale that satisfy these queries. Two of the three Nissans satisfy the first query, and all the Nissans and the Mitsubishi, but not the Toyota, satisfy the second query. Unfortunately, however, search engines do not access the facts within these ads in the way we would wish to find these cars. Our approach of superimposing a web of knowledge over a web of pages makes these facts visible from outside the page and directly accessible to query engines (as opposed to search engines).

To make this work, we need an ontology for car ads. Figure 15.2 shows an example. The ontology is a conceptual model. It consists of object sets which are either lexical (dashed boxes in Figure 15.2) or non-lexical (solid boxes). Instances in lexical object sets are strings of characters such as 'Nissan' or '1990'; whereas instances in non-lexical object sets are object identifiers that stand for real-world objects-Car ${ }_{73}$ and $\mathrm{Car}_{1194}$, for example, identify specific cars. Relationship sets in the conceptual model are lines connecting object sets. Min-max participation constraints impose restrictions on the relationship sets: a car described in a car ad can have zero or more features $\left(0:^{*}\right)$, but at most one make $(0: 1)$. Ontologies for our WoK vision also support hypernym-hyponym is-a hierarchies and holonym-meronym part-of hierarchies. A white triangle denotes an is-a hierarchy, so that, for example, a Body Type is-a Feature in Figure 15.2. The plus symbol (+) in the triangle specifies a disjointness constraint among the specializations; it is also possible to declare union constraints $(\cup)$ specifying that a generalization object set is a union of its specialization object sets and partition constraints $(\uplus)$ specifying that specialization object sets partition their generalization. A black triangle denotes a part-of hierarchy. In Figure 15.2, for example, a model such as "Accord" aggregated with a trim specification such as "LX" constitutes the concept "Accord LX", which has some ModelTrim object identifier (e.g., ModelTrim 39 ).

Whereas an ontology tells us what kind of knowledge exists in our domain of interest, epistemological specifications tell us what the knowledge is and how it is acquired. For our WoK vision, populating an ontology yields knowledge. We can populate the the car-ad ontology in Figure 15.2 


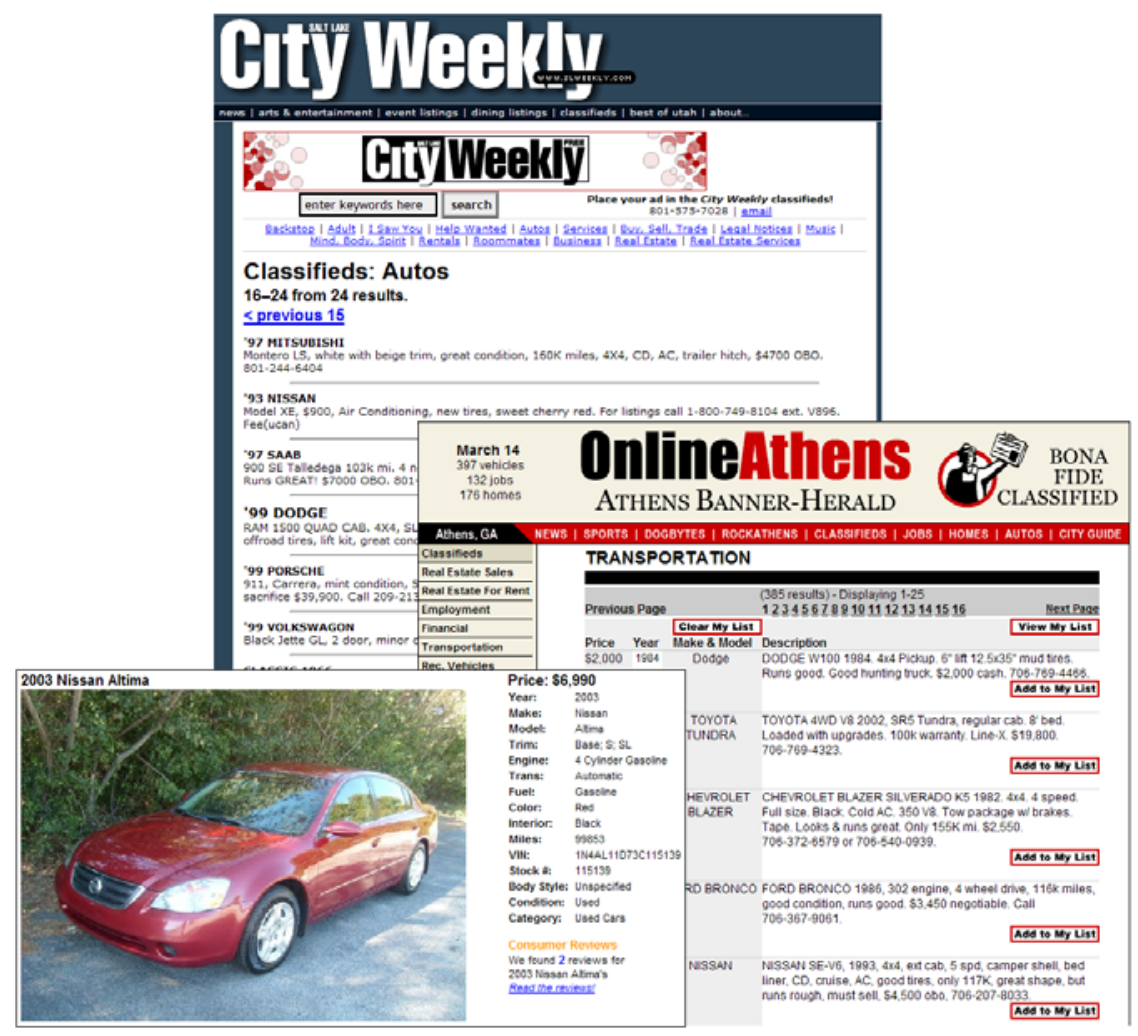

Fig. 15.1 Sample Car Ad Web Pages.

with facts derived from car ads. Assuming $\mathrm{Car}_{73}$ denotes the Nissan Al-

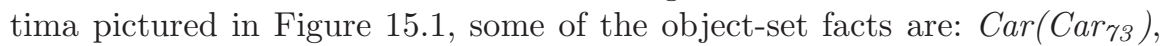
Year(2003), and Transmission("Automatic"), and some of the relationship facts are: CarYear(Car ${ }_{73}$, 2003) and CarFeature(Car ${ }_{73}$, "Automatic").

The way we intend to acquire knowledge in our WoK vision is particularly interesting. Although possible to simply encode by hand, this is far too labor intensive and does not scale. We must find ways to automatically identify facts and associate them with ontologies. Sometimes information is structured in such a way that it is possible to reverse-engineer it into an ontology; sometimes it is possible to resort to available outside knowledge sources to align semi-structured information with an ontology; but sometimes the information yields to neither of these techniques. For this latter case, we augment the ontologies themselves so that they are capable of recognizing, annotating, and extracting relevant facts with respect to their ontological descriptions. We call these augmented ontologies extraction ontologies, because they are 


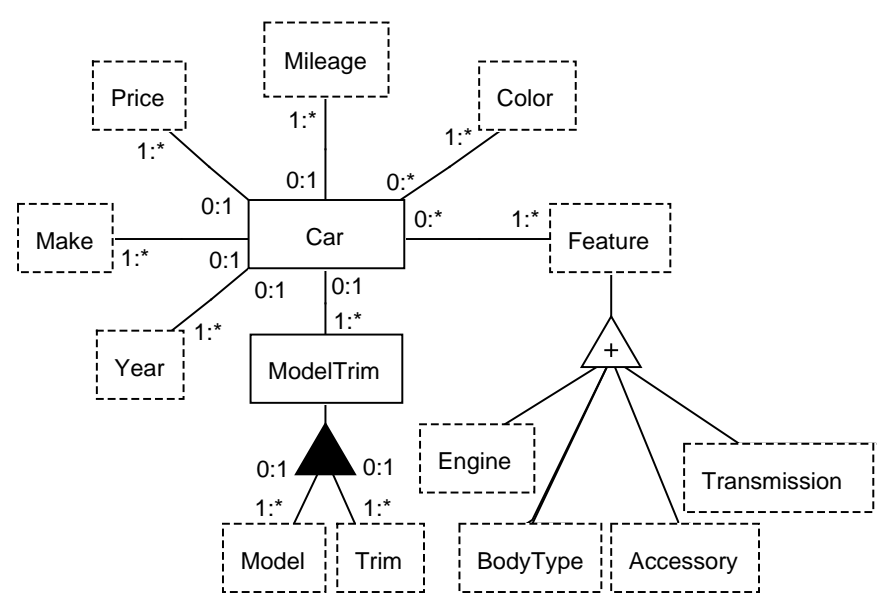

Fig. 15.2 Car Ad Ontology.

capable of locating and extracting facts from any kind of document, unstructured as well as semi-structured and structured.

The augmentation that turns an ontology into an extraction ontology is a data frame [Emb80]. Data frames are linguistically grounded abstract data types - they encapsulate everything we wish to know about categorized data instances including their internal and external representations and their applicable operations. The linguistic grounding consists of providing recognizers for instances of object and relationship sets. Figure 15.3 shows examples of the kind of recognizers we currently use in our WoK vision, but any kind of information extractor [Sar08] is possible: e.g., machine-learned wrappers (e.g., [WCW ${ }^{+}$09]), NLP-based recognition techniques (e.g., [FM09]), and elaborate handcrafted rules (e.g., [GGTB08, $\left.\mathrm{HLF}^{+} 08\right]$ ). The recognizers in Figure 15.3 are of two types - regular expressions and lexicons - which operate independently or together. The regular expression for the external representation of Price in Figure 15.3 recognizes price instances such as "\$23,900" and " 15 grand". The external-representation recognizer for Make, on the other hand, is a lexicon that lists all the makes of cars, including their alternate spellings and abbreviations. Context keywords such as price and asking in Figure 15.3 help disambiguate instances that may be recognized for more than one concept (e.g., MSRP price vs. asking price). Operators also have recognizers. Keywords such as "less than", "<", and "under" in Figure 15.3 indicate the applicability of the LessThan operator. A price instance following these keywords becomes the second parameter $p_{2}$ in the operation, the car price being assumed as the first.

Justification of captured knowledge is a natural consequence of acquiring and semantically annotating knowledge. When we reverse engineer structured 


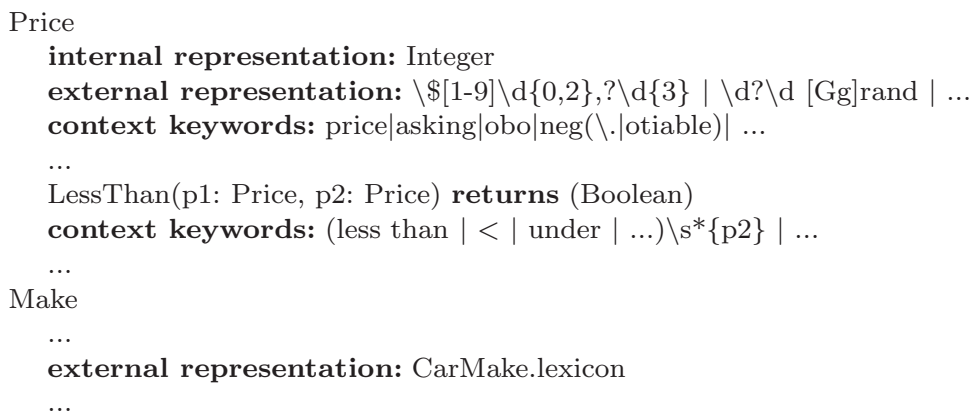

Fig. 15.3 Data Frames.

knowledge into a fact-filled ontology, align facts in a knowledge source with an ontology, or extract facts from data-rich documents, the WoK system keeps track of the source of each fact. Later, when someone queries for these facts, the WoK system provides, in addition to the standard query results, a cached page with the fact instances highlighted. For example, if we query for a red Nissan Altima for under 15 grand, as part of the answer the WoK system can retrieve the cached page of the Altima in Figure 15.1 and display it with the strings Red, Nissan, Altima, and \$6,990 highlighted.

Besides enabling fact recognition in source documents, extraction ontologies also enable free-form query processing. For example, a WoK system with the ontology in Figure 15.1 augmented with the data-frame recognizers in Figure 15.3 can interpret and process the query: "Find me a red Nissan Altima for under 15 grand." The system associates the recognized instances "red", "Nissan", "Altima", and "15 grand" respectively with the object sets Color, Make, Model, and Price, and it associates "under" with the LessThan operator in the Price data frame and the price " 15 grand" with the operator's parameter $p 2$. Generating a formal select-project-join query from these recognized associations is straightforward: do outer join over the ontology's structure; select based on the identified constants and Boolean operations; and project on the mentioned object sets.

Retrieving inferred facts is more complex. To illustrate, consider processing the query, "I'd like a Japanese-made car for under 15 grand." For this query the WoK system needs a way to determine which cars are Japanese. Suppose we have a second ontology about car manufacturers such as the one in Figure 15.4. Observe that the concept Make in Figure 15.1 is semantically the same as the concept Make in Figure 15.4. Connecting these concepts with a between-ontologies same-as link is an example of the interconnecting links in the web-like structure constituting a WoK. With these two ontologies and the interconnecting link, we now have the information we need to declare inference rules that can reason about a car being Japanese-made. A car in a car ad that has a make produced by a manufacturer whose headquarters is in Japan is a Japanese-made car. 


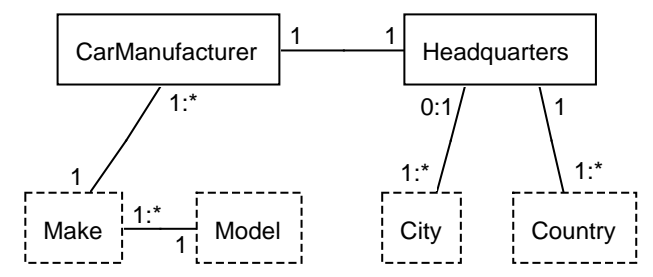

Fig. 15.4 Car Manufacturer Ontology.

Next observe that populated ontologies are in reality first-order-language theories of predicate calculus. Each object set $S$ is a one place predicate $S(x)$. Each $n$-ary relationship set $R$ is an $n$ place predicate, $R\left(x_{1}, \ldots, x_{n}\right)$. The constraints of our conceptual-modeling language are all expressible as closed well-formed formulas of predicate calculus. Populating an ontology provides the ground facts for a first-order theory. When all the constraints hold for a populated ontology, we have a model of the first order theory. And, we can reason over the model with logic languages, appropriately restricted to make them decidable and tractable.

For our query about Japanese-made cars, suppose we have the following logic rule (expressed in Datalog-like syntax) for the car-manufacturer ontology:

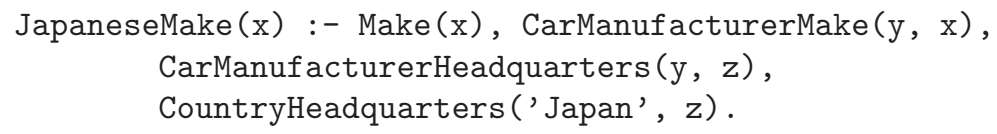

Similar to linguistically grounding operators in our ontologies, we can linguistically ground logic rules. Thus, the phrase "Japanese-made car" should indicate that this rule applies. Now, when a user poses a query for Japanesemade cars for under 15 grand, the WoK system recognizes that the two ontologies apply and generates the following formal query over the two theories CarAd and CarManuf:

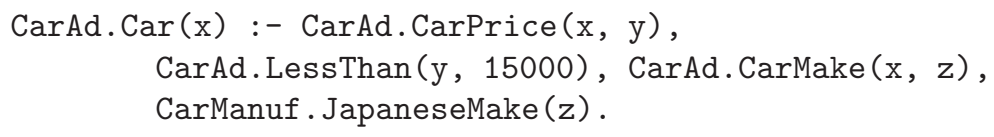

Here, the same-as link lets us seamlessly navigate among populated ontologies in a WoK. As an aside, note the conversion of " 15 grand" to the integer 15000. As indicated in the Price data frame in Figure 15.3, the type for the internal representation is integer. As the WoK system extracts data for an ontology, it also converts the data to declared internal representations.

By way of summary for this informal introduction to our WoK vision, we see a WoK consisting of logic theories, interconnected and superimposed over web documents. Logic theories are populated ontologies. From an epistemological point of view, populated ontologies are extensional knowledge. 
And with the addition of inference rules, populated ontologies also embody intensional knowledge.

\subsection{WoK Formalization}

We base our foundational conceptualization for a web of knowledge on the conceptual modeling language OSM (Object-oriented Systems Modeling) [EKW92]. OSM, however, simply provides a graphical representation of a first-order-logic language. Here we restrict OSM to be decidable, yet powerful enough to represent desired ontological concepts and constraints. We call our restriction OSM-O, short for OSM-Ontology. We thus base our foundational conceptualization directly on an appropriate restriction of first-order logic. This WoK foundation should be no surprise since it is the basis for modern information systems and has been the basis for formalizing information since the days of Aristotle [AriBC].

Definition 1. OSM- $O$ is a triple $(O, R, C)$ :

- $O$ is a set of object sets; each is a one-place predicate; and each predicate has a lexical or a non-lexical designation.

- $R$ is a set of $n$-ary relationship sets $(n \geq 2)$; each is an $n$-place predicate.

- $C$ is a set of constraints:

- Referential integrity: $\forall x_{1} \ldots \forall x_{n}\left(R\left(x_{1}, \ldots, x_{n}\right) \Rightarrow S_{1}\left(x_{1}\right) \wedge \ldots \wedge S_{n}\left(x_{n}\right)\right.$ for each $n$-ary relationship set $R$ connecting object sets $S_{1}, \ldots, S_{n}$.

- Participation constraint min:max cardinality: for every connection of an object set $S$ to an $n$-ary relationship set $R, \forall x_{i}\left(S\left(x_{i}\right) \Rightarrow \exists^{\geq{ }^{m i n}}<x_{1}\right.$, $\left.\ldots, x_{i-1}, x_{i+1}, \ldots, x_{n}>\left(R\left(x_{1}, \ldots, x_{n}\right)\right)\right)$ if $\min >0$, and $\forall x_{i}\left(S\left(x_{i}\right) \Rightarrow\right.$ $\left.\exists \leq \max <x_{1}, \ldots, x_{i-1}, x_{i+1}, \ldots, x_{n}>\left(R\left(x_{1}, \ldots, x_{n}\right)\right)\right)$ if $\max$ is not $*$ (the symbol denoting an unbounded maximum).

- Generalization/specialization: $\forall x\left(S_{1}(x) \vee \ldots \vee S_{n}(x) \Rightarrow G(x)\right)$ for each generalization object set $G$ of specialization object sets $S_{1}, \ldots, S_{n}$ in a hypernym-hyponym is-a hierarchy. In addition, $\forall x\left(S_{i}(x) \Rightarrow \neg S_{j}(x)\right)$ for $1 \leq i, j \leq n$ and $i \neq j$ if the specialization object sets are disjoint and $\forall x\left(G(x) \Rightarrow S_{1}(x) \vee \ldots \vee S_{n}(x)\right)$ if the generalization object set is complete - is a union of the specialization object sets.

- Aggregation: holonym-meronym relationship sets grouped as an aggregation in an is-part-of hierarchy.

Example 1. Figure 15.2 shows an OSM-O model instance. Car $(x)$ and Model$\operatorname{Trim}(x)$ are the one-place predicates for the two non-lexical object sets. Mileage $(x)$ and Engine $(x)$ are two of the one-place predicates for the lexical object sets. CarYear $(x, y)$ is a two-place predicate for the relationship set connecting $C a r$ and $Y$ ear. For readability, we may provide a more descriptive name for a relationship set so long as the naming phrase for the rela- 
tionship set includes the names of its object sets. We then typically use infix notation and write, for example, Car(x)hasYear(y) for the CarYear $(x, y)$ relationship set or Car $(x) \operatorname{costsPrice}(y)$ for the CarPrice $(x, y)$ relationship set. One of the referential integrity constraints is $\forall x \forall y(C a r Y e a r(x, y) \Rightarrow$ $\operatorname{Car}(x) \wedge Y e a r(y))$. One of the participation constraints is $\forall x(\operatorname{Car}(x) \Rightarrow$ $\exists \leq 1 y(\operatorname{CarY} \operatorname{ear}(x, y)))$, where we drop the tuple-grouping angle brackets for the common case of only one variable being existentially quantified. The formula $\forall x(\operatorname{Engine}(x) \vee \operatorname{BodyType}(x) \vee \operatorname{Accessory}(x) \vee \operatorname{Transmission}(x) \Rightarrow$ Feature $(x)$ ) defines the generalization/specialization; its disjointness constraint includes $\forall x($ Engine $(x) \Rightarrow \neg \operatorname{BodyType}(x))$ as one of its terms. An aggregation groups several relationship sets denoting subparts of superparts: Model(x)isSubpartOf ModelTrim(y) and Trim(x)isSubpartOfModelTrim $(y)$ are the two relationship sets of the aggregation in Figure 15.2; their inverses are respectively ModelTrim(x)isSuperpartOfTrim(y) and ModelTrim(x)isSuperpartOfModel(y). Although graphical in appearance, an OSM-O diagram is merely a two-dimensional rendition of predicates and closed formulas as defined in Definition 1.

Definition 2. Let $M=(O, R, C)$ be an OSM-O model instance. Let $I$ be an interpretation for $M$ that has a domain $D=L_{I D} \cup O_{I D}$, where $L_{I D} \cap O_{I D}=$ $\varnothing$, and a declaration of True or False for each valid instantiation of each predicate in $O \cup R$. For predicates in $O$, valid instantiations require lexical predicates to be instantiated with values in $L_{I D}$ and non-lexical predicates to be instantiated with values in $O_{I D}$. For predicates in $R$, valid instantiations require each value $v$ to be lexical or non-lexical according to whether the connected object set for $v$ is lexical or non-lexical respectively. If all the constraints of $C$ hold, $I$ is a model of $M$, which we call a valid interpretation of $M$ (to avoid an ambiguous use of the word "model" when also discussing conceptual models). An instantiated True predicate for a valid interpretation is a fact.

Example 2. A valid interpretation of the OSM-O model instance in Figure 15.2 contains facts about cars. A valid interpretation might include the facts $C a r\left(C_{3}\right.$ ), Year(2003), CarY ear(Car 3,2003$)$, Model ("Accord"), Trim("LX"), ModelTrim(ModelTrim 17 ), CarModelTrim(Car 3 , ModelTrim $_{17}$ ), and Trim( "LX")isPartOfModelTrim(ModelTrim ${ }_{17}$ ). The object sets Car and ModelTrim, being non-lexical, have object identifiers for their domain-value substitutions (which we denote by object-set names with a subscript). The constraint $\forall x\left(\operatorname{Car}(x) \Rightarrow \exists^{\leq 1} y(\operatorname{CarY} \operatorname{ear}(x, y))\right)$ holds for $\mathrm{Car}\left(\mathrm{Car}_{3}\right)$ if $\mathrm{CarY}$ ear $\left(\mathrm{Car}_{3}, 2003\right)$ is the only car-year pair that exists with $\mathrm{Car}_{3}$ as its first element.

Similar to the work by Buitelaar, et al. [BCHS09], we now show how to linguistically ground OSM-O. Linguistically grounding OSM-O turns OSMO model instances into OSM-Extraction-Ontology model instances (OSM-EO model instances). We begin by defining an ordinary abstract data type for 
each object set and relationship set. We then add linguistic recognizers for instance values, operators, operator parameters, and relationships.

Definition 3. An abstract data type is a pair $(V, O)$ where $V$ is a set of values and $O$ is a set of operations.

Definition 4. A data frame is an abstract data type augmented as follows:

1. The data frame has a name $N$ designating the set of values $V$, and it may have a list of synonyms for $N$.

2. The value set $V$ has instance recognizers that identify lexical patterns denoting values in $V$.

3. For a lexical object set, the operator set $O$ includes input operators to convert identified instances to an internal representation and output operators to convert the internal representation of instances to displayable strings.

4. An operation $o$ in $O$ may have a recognizer that identifies lexical patterns in text that indicate that $o$ applies. Further, the recognizer identifies lexical patterns that, along with instance recognizers, identify parameters for $o$.

Example 3. In Figure 15.3 the value set $V$ for the Price data frame is of type Integer. Its recognizer is a potentially lengthy list of regular expressions augmented by keywords. Its operation set $O$ includes the LessThan operator and potentially has many more operations. The LessThan operator has keyword phrases that indicate its applicability as well as how to identify its parameters.

For relationship sets, the definition of a data frame does not change, but a typical view of the definition shifts as we allow value sets to be $n$-tuples of values rather than scalar values. Further, like recognizers for operators, they rely on instance recognizers from the data frames of their connected object sets.

Example 4. Suppose the Car object set in Figure 15.2 has a relationship set to a Person object set. The relationship-set data frame may have recognizers for any one of several possible relationships such as $\{$ Person $\}$ is selling $\{$ Car $\}$, $\{$ Person $\}$ posted $\{C a r\}$ ad, or $\{$ Person $\}$ is inquiring about $\{C a r\}$ Here, the braces enclose references to data frames for the non-lexical object sets Car and Person.

As is standard, implementations of abstract data types are hidden, and we hide implementations for data frames as well. Similar to data independence in database systems, this approach accommodates any implementation. In particular, it allows for new and better recognizers, which we can draw from the large body of work devoted to information extraction [Sar08].

Definition 5. If $M$ is an OSM-O model instance with a data frame for each object set and relationship set, $M$ is an OSM-EO model instance. 
An OSM-EO model instance is linguistically grounded in the sense that it can both "read" and "write" in some natural language. To "read" means to be able to recognize facts in natural language text and to extract fact instances with respect to the ontology in the OSM-EO model instance. To "write" means to display fact instances so that they are human-readable.

How well a particular OSM-EO model instance can "read" and "write" makes a difference in how well it performs. Our experience is that OSM-EO model instances can "read" some documents well (over 95\% precision and recall [ECJ+99]), but it is clear that opportunities abound for further research and development. Writing human-understandable descriptions is less difficult to achieve - just select any one of the phrases for each object set and relationship set (e.g., Person(Person 17$)$ is selling $\operatorname{Car}\left(\right.$ Car $\left._{734}\right)$, Car $_{\left(\text {Car }_{734}\right)}$ has Make(Honda)). Making written descriptions more pleasing, of course, is more difficult.

Continuing in our quest to define the components of our WoK vision, we now define its "knowledge," and we explain how we see its "knowledge" being justified and how we envision its knowledge components being interconnected.

Definition 6. The collection of facts in an OSM-O model instance constitutes its extensional knowledge. The collection of implied facts derived from the extensional knowledge by inference rules ${ }^{2}$ constitutes its intensional knowledge. The extensional and intensional knowledge together constitute the knowledge of the OSM-O model instance.

Although this view of knowledge is common in computing, Plato, and those who follow his line of thought, also demand of knowledge that it be a "justified true belief" [PlaBC]. "Knowledge" without some sort of truth authentication can be unsupported and even misleading. For our vision of a WoK, we attempt to establish truth via provenance and authentication. When an extraction ontology extracts a fact from a source document, it retains a link to the fact; and when a query answer requires reasoning over rules, the system records the reasoning chain. Users can ask to see fact sources and rule chains, and in this way they can authenticate facts and reasoning the way we usually do-by checking sources and fact-derivation rules.

Definition 7. A knowledge bundle $(K B)$ is a 5-tuple $(O, E, S, I, R)$ where $O$ is an OSM-O model instance; $E$ is an OSM-EO instance whose OSM-O instance is $O ; S$ is a set of source documents from which facts for $E$ are extracted; $I$ is a valid interpretation for $O$ whose facts are extracted from the documents in $S$; and $R$ is a set of inference rules.

Finally, to make the envisioned WoK truly a web of knowledge, we interconnect knowledge bundles (KBs). Facts about the same object may appear

2 As the work on logic and particularly on description logics [BN03] continues to expand, we can take advantage of the work of this community (e.g., [Ros05, CGL09, CGP09]) to employ better and more powerful reasoning engines. 
in more than one KB. We can directly connect these objects so that users may navigate among KBs and obtain additional information about an object. Concepts in more than one KB may also be essentially the same as is the concept Make, and also the concept Model, in their respective ontologies in Figures 15.2 and 15.4. We also connect concepts across ontologies to provide additional navigation paths.

Definition 8. A Web of Knowledge (WoK) is a collection of knowledge bundles interconnected with binary links, $\langle x, y\rangle$, of two types: (1) object identity: non-lexical object identifier $x$ in knowledge bundle $B_{1}$ refers to the same real-world object as non-lexical object identifier $y$ in knowledge bundle $B_{2}$. (2) Object-set identity: object set $x$ in knowledge bundle $B_{1}$ designates the same kind of real-world objects as object set $y$ in knowledge bundle $B_{2}$.

\subsection{WoK Construction}

To construct a WoK, we must be able to construct a knowledge bundle (KB), and we must be able to establish links among KBs. We can construct KBs and establish links among them by hand (and this should always be an option). However, scaling WoK construction demands semi-automatic procedures, with much of the construction burden placed on the system-all of it when possible. Our KB construction tools transform, or aid in transforming, source information into KB components. For links among KBs we apply record-linkage and schema-mapping tools.

Definition 9. A transformation is a 4 -tuple $(R, S, T, \Sigma)$, where $R$ is a set of resources, $S$ is the source conceptualization, $T$ is the target conceptualization for an $S$-to- $T$ transformation, $\Sigma$ is a set of source-to-target transformation statements.

Definition 9 leaves several of its components open - to take on specific meanings in a variety of KB building tools. The "set of resources" is undefined, but we intend this to mean semantic resources such as WordNet and a data-frame library. "Target conceptualizations" are KBs or KB components. "Source conceptualizations" depend on sources whose fact conceptualizations can be formal, semi-formal, or informal. "Source-to-target transformation statements" can be declarative or procedural and can be written in a variety of formal languages.

To the extent possible, we want our transformations to preserve the information and constraints in source documents and repositories. When sources are formalized as predicate calculus or in a formalization equivalent to predicate calculus, we can guarantee the preservation information and constraints. We identify the predicates and the facts for the predicates (thus preserving information) and formulate a closed well-formed formula for each constraint 
(thus preserving constraints). If the source interpretation is valid, the target interpretation will be valid as well. When sources are informal with respect to predicate calculus, the predicates, facts for the predicates, and the constraints are implicit. The challenge is nevertheless to discover and extract them.

Definition 10. Let $S$ be a predicate calculus theory with a valid interpretation, and let $T$ be a populated OSM-O model instance constructed from $S$ by a transformation $t$. Transformation $t$ preserves information if there exists a procedure to compute $S$ from $T$. Let $C_{S}$ be the closed, well-formed formulas of $S$, and let $C_{T}$ be the closed, well-formed formulas of $T$. Transformation $t$ preserves constraints if $C_{T} \Rightarrow C_{S}$.

Our goal has been and is to successfully develop automatic and good semiautomatic transformations over a broad spectrum of documents for a variety of ontological contexts. For sources whose facts and constraints over these facts have formal declarations, transformations should preserve all facts and constraints. For sources whose facts and constraints are implicit, we seek to identify the facts and constraints that are applicable to a given ontology or, in the absence of a given ontology, we seek to determine and populate the implicit ontology based on the document's data and on applicable external knowledge resources.

Longstanding research endeavors can all contribute to various parts of WoK construction. These include: reverse engineering [Aik98], table and form understanding [EHLN06, dSJT06], ontology learning [Cim06], ontology alignment [ES07], data integration [BN08, HRO06, RB01], and record linkage [EIV07]. In Sections 15.4.1-15.4.4 we explain how we have taken advantage of some of this work for WoK construction. In Section 15.4.1 we show how to reverse engineer XML data repositories into KBs. In Section 15.4.2 we describe how we can interpret collections of nested tables in hidden web pages and thus turn the collection into a KB. In Section 15.4.3 we explain how we integrate a group of semantically overlapping tables to create a KB. And, in Section 15.4.4 we give a way via form creation and information harvesting to generate KBs. Finding and implementing other ways to construct WoK components are interesting and worthwhile research endeavors.

\subsubsection{Construction via XML Reverse Engineering}

We have designed a conceptual model for XML, called C-XML (Conceptual XML). C-XML adds a few XML-specific concepts to OSM-O, including in particular a sequencing construct and a choice construct. Being formally defined as templates over OSM-O constructs, however, these additions remain within the purview of OSM-O. 
Reverse engineering an XML schema to C-XML effectively defines a mapping to OSM-O for all XML documents complying to the XML schema [AK07]. The basic translation strategies for mapping XML Schema to C-XML are straightforward, although some parts of the translation require some sophisticated manipulation. In the translation, elements and attributes become object sets. Elements that have simple types become lexical object sets, while elements that have complex types become non-lexical object sets. Attributes become lexical object sets since they always have a simple type. Built-in data types and simple data types for an element or an attribute in XML Schema are specified in the data frame associated with the object set representing the element or the attribute. XML parent-child connections among elements and XML element-attribute connections both become binary relationship sets in C-XML. The constraints minOccurs and maxOccurs translate directly to participation constraints in C-XML.

Unfortunately, not everything is straightforward. Translations for keys, extension, restriction, substitution groups, and mixed content are all quite interesting. The translation also involves a myriad of detail extending to over 40 pages in [AK07]. Although extensive, the translation details provide a constructive proof that the transformation from XML Schema to C-XML preserves both information and constraints.

The result of doing information- and constraint-preserving transformations of XML documents complying to an XML schema is a KB. Further, to the extent that we can automatically infer an XML schema specification directly from an XML document, we can also reverse-engineer raw XML documents into populated C-XML model instances and thus into KBs.

\subsubsection{Construction via Nested Table Interpretation}

TISP (Table Interpretation with Sibling Tables) is a tool of ours that interprets tables in sibling pages [Tao08]. To interpret a table is to properly associate table category labels with table data values. Using Figure 15.5 as an example, we see that Identification, Location, and Function are labels for the large rectangular table. Inside the cell labeled Identification is another table with headers IDs, NCBI KOGs, Species, etc. Nested inside of this table are two more tables, the first starting with the label $C G C$ name and the second starting with the label Gene Model. We associate labels with data values by observing the table structure. A cell in a table associates with its header label (or labels in the case of multi-dimensional tables). For nested tables, we trace the sequence of labels from the outermost label to the data cell. Thus, for example, the label for the value F47G6.1 under Sequence name is Identification.IDs.Sequence_name.

Although automatic table interpretation can be complex, if we have another page, such as the one in Figure 15.6, that has essentially the same 


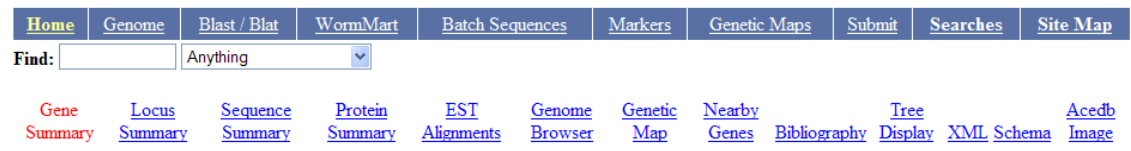

\section{Gene Summary for dyb-1}

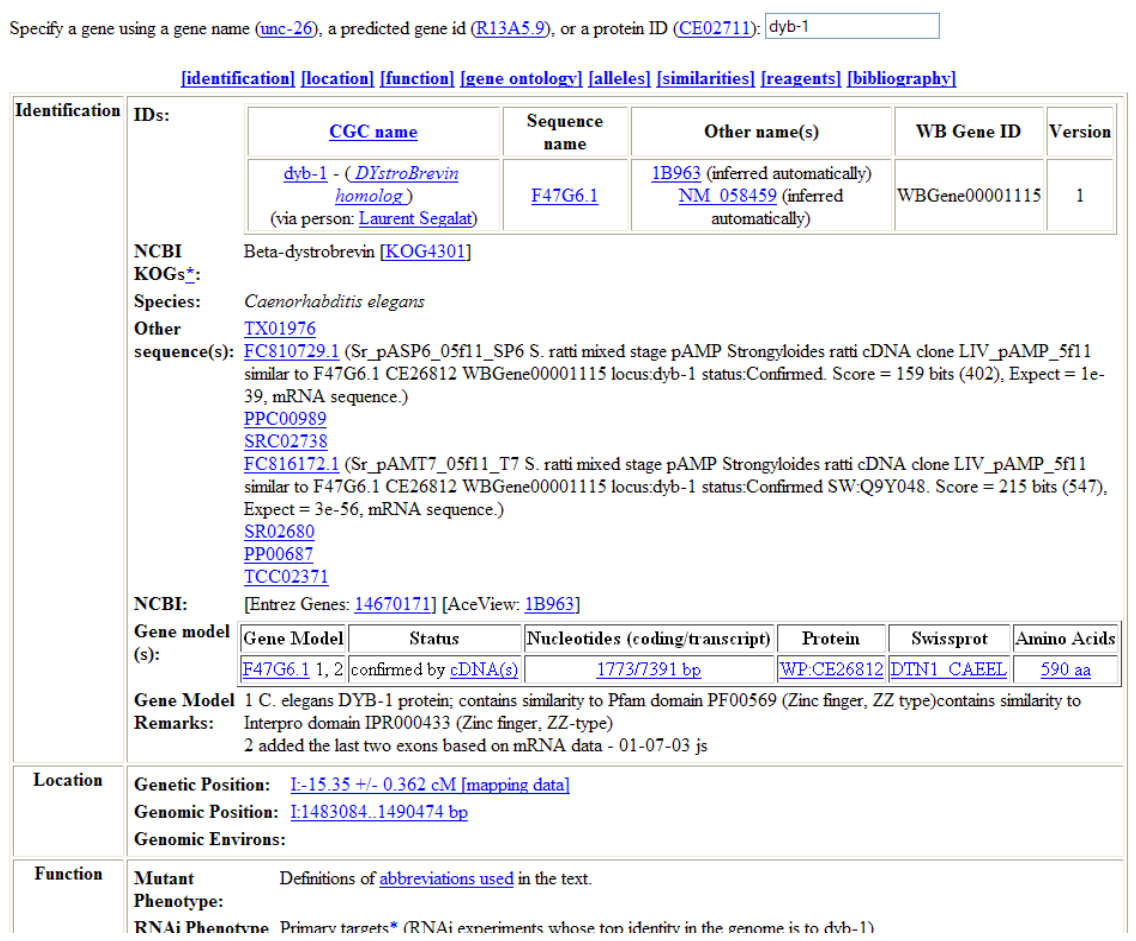

Fig. 15.5 Nested Table from a Molecular-Biology Web Page.

structure, the system can usually obtain enough information to make automatic interpretation possible. We call pages that are from the same web site and have similar structures sibling pages. The two pages in Figures 15.5 and 15.6 are sibling pages. They have the same basic structure, with the same top banners that appear in all the pages from this web site, with the same table title (Gene Summary for some particular gene), and a table that contains information about the gene. Corresponding tables in sibling pages are called sibling tables. If we compare the two large tables in the main part of the sibling pages, we can see that the first columns of each table are exactly the same. If we look at the cells under the Identification label in the two tables, both contain another table with two columns. In both cases, the first column contains the identical labels IDs, ..., Remarks, although the table in Figure 15.6 has one additional label, Notes. Further, the tables under Identification.IDs also have identical header rows, and the tables under Iden- 


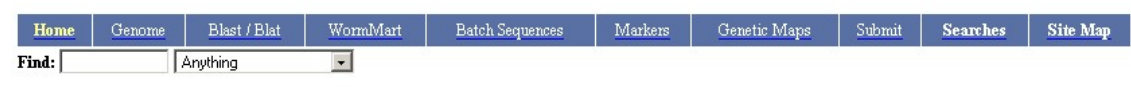

\section{Gene Summary for cdk-4}

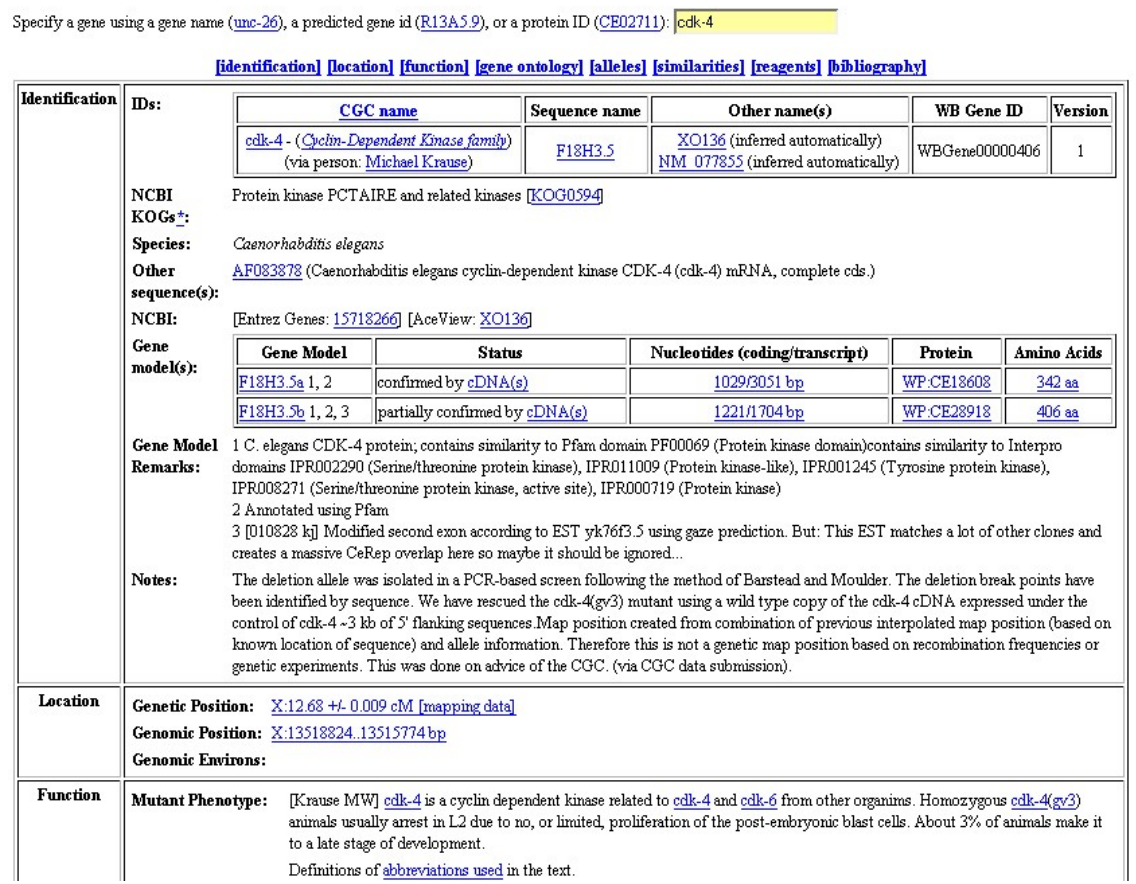

Fig. 15.6 Sibling Page of the Page in Figure 15.5.

tification.Gene model(s) have nearly identical header rows. The data values, however, vary considerably. Generally speaking, we can look for commonalities in sibling tables to find labels and look for variations to find data values.

Given that we can interpret a table - find labels and values and properly associate them - we can create a conceptualization of the table linking labels as metadata with values as instance data. This simple conceptualization may not always be best, but for some tables it works well. In particular, it works well for nested tables like the ones in Figures 15.5 and 15.6.

Observe that for these nested tables, the conceptual, nested, label-value structure is isomorphic to a simple XML schema. There exists a single, nested, label path to every data value. For the sequence name $F 47 G 6.1$ in the top row of the table in Figure 15.5, the label path is Identification.IDs.Sequence_name. This nested-label property lets us conceptualize these tables in an XML-like conceptual tree with labels as tags and instance values as leaf strings in a nested tag structure, as Figure 15.7 illustrates. Note that the tree structure in Figure 15.7 precisely captures the nesting of the tables in Figures 15.5 
and 15.6. Note also that the conceptualization can account for the variation among tables. In Figure 15.7, for example, Gene Models relates optionally to Swissprot because the ontology-generation process observes that one table (Figure 15.5) has an entry for Swissprot whereas the other (Figure 15.6) does not. The end result is an automatically generated KB containing all the data from the given set of sibling tables.

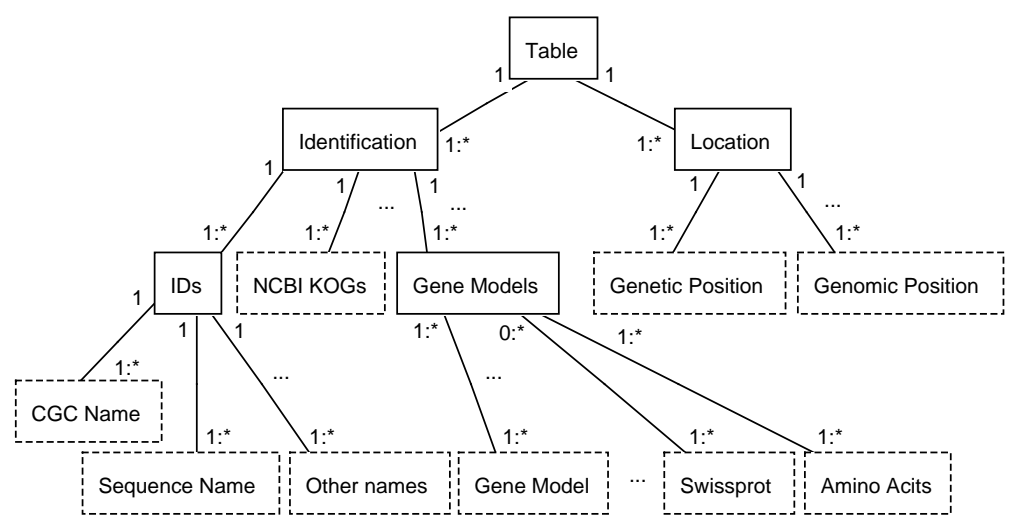

Fig. 15.7 Generated Conceptual Model Instance.

\subsubsection{Construction via Semantic Integration}

TANGO (Table ANalysis for Generating Ontologies) provides another way to create KBs. Given a collection of tables all in the same application domain, we reverse-engineer each table into a conceptual-model instance and then integrate the conceptual-model instances into an ontology that represents the domain. During the process, we analyze each table individually, inferring concepts, relationships among concepts, and data values for concepts and relationships. The result of this process is a conceptual-model instance for the table, which we call a "mini-ontology" - "mini" because the number of concepts in a table is usually small. We then exploit schema-mapping techniques to discover interrelationships among the mini-ontologies, enabling us to merge the generated conceptual-model instances into an ontological structure for the domain.

TANGO operates in three steps:

1. Recognize and canonicalize table information.

2. Construct mini-ontologies from canonicalized tables. 


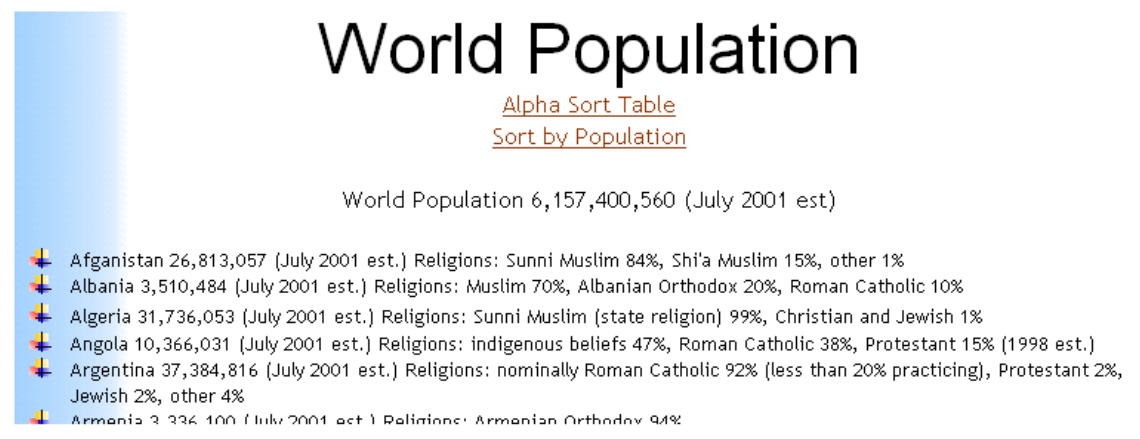

Fig. 15.8 World Populations and Religions.

3. Discover inter-ontology mappings and merge mini-ontologies into a growing application ontology.

We illustrate with an example.

\subsubsection{Table Recognition and Canonicalization}

Tables appear in many shapes and sizes; most, but not all, have rectangular grid layouts. Figure 15.8 is an example of a table without a clearly delineated grid layout.

In our first step, we canonicalize tables by converting them to "Wang notation," a layout-independent formalization for tables [Wan96]. Wang notatation has two parts: (1) label structure and (2) data-value/label-structure association. Label structure consists of a collection of dimension trees, one for each coordinate that indexes a data cell. A dimension tree organizes labels in a tree structure: each path from root to leaf provides an index coordinate for a data value. In our example, the table in Figure 15.8 has two dimensions. In Wang notation, these two dimension trees are: 
(DT1Root, \{

(Population (July 2001 est.), Ø), (Religion, \{

(Albanian Orthodox, $\varnothing$ ), (Muslim, Ø),

(Roman Catholic, Ø), (Shi'a Muslim, Ø), (Sunni Muslim, Ø), ...

(other, Ø)

\}))

(Country, \{

(Afghanistan, Ø), (Albania, Ø),

\})

Wang associates data with dimension trees in $\delta$-statements. Each combination of paths through dimension trees can have a value:

$\delta$ (DT1Root.Population (July 2001 est.), Country.Afghanistan $)=26,813,057$

$\delta($ DT1Root.Religion.Albanian Orthodox, Country.Afghanistan $)=\perp$

..

$\delta($ DT1Root.Religion.other, Country.Afghanistan $)=1 \%$

$\delta$ (DT1Root.Population (July 2001 est.), Country.Albania) $=3,510,484$

...

Here, the first statement is for the data cell containing $26,813,057$, the population of Afghanistan. The other three index an empty cell, the $1 \%$ for "other" religions in Afghanistan, and the 3,510,484 for the population of Albania. Similarly, we can index all values for all countries in Figure 15.8.

We consider any collection of data that we can represent in Wang notation to be a table. Further, given a table in Wang notation, we can display the table in a standard grid form. We place the first dimension above the data, the second to the left of the data, the third to the left of the second with the second replicated for every leaf of the third, .... We omit implicit roots, such as the root DT1Root for Dimension Tree 1. Figure 15.9 displays the table in Figure 15.8 in this standard way. Figures 15.10-15.14 show several additional examples, which together with Figure 15.8 constitute, for our example here, the tables to be merged into a KB.

We [EHLN06, JNS ${ }^{+}$09, TE09], and others (e.g., [GBH ${ }^{+}$07, $\mathrm{PSC}^{+}$07, RK06, dSJT06, ZBC04]), are working toward fully automatic table-interpretation tools. These tools take as input a table such as the one in Figure 15.8, and produce as output Wang notation, which we can display in a standard way. In our tools, we augment Wang notation so that it can capture more than just labels and values. We also capture a table's title, its footnotes, and its units of measure. In the absence of fully automated tools, we have developed 


\begin{tabular}{|c|c|c|c|c|c|c|c|}
\hline \multirow[b]{2}{*}{ Country } & \multirow[b]{2}{*}{$\begin{array}{c}\text { Population } \\
\text { (July } 2001 \text { est.) }\end{array}$} & \multicolumn{6}{|c|}{ Religion } \\
\hline & & \begin{tabular}{|l} 
Albanian \\
Orthodox
\end{tabular} & Muslim & $\begin{array}{c}\text { Roman } \\
\text { Catholic }\end{array}$ & \begin{tabular}{|l} 
Shi'a \\
Muslim
\end{tabular} & $\begin{array}{l}\text { Sunni } \\
\text { Muslim }\end{array}$ & other \\
\hline $\begin{array}{l}\text { Afghanistan } \\
\text { Albania }\end{array}$ & $\begin{array}{r}26,813,057 \\
3,510,484\end{array}$ & $20 \%$ & $70 \%$ & $10 \%$ & $15 \%$ & $84 \%$ & $1 \%$ \\
\hline
\end{tabular}

Fig. 15.9 Canonicalized Table for World Religious Populations.

\begin{tabular}{|c|c|c|c|c|c|}
\hline \multirow[b]{2}{*}{ Country } & \multirow{2}{*}{$\begin{array}{c}\text { Population } \\
\text { (July } 2003 \text { est.) }\end{array}$} & \multicolumn{3}{|c|}{ Median Age (2002) } & \multirow{2}{*}{$\begin{array}{c}\text { Population Growth Rate } \\
\text { (2003 est.) }\end{array}$} \\
\hline & & Total & Male & Female & \\
\hline Afghanistan & $28,717,213$ & 18.9 years & 19.1 years & 18.7 years & $3.38 \% *$ \\
\hline Albania & $3,582,205$ & 26.5 years & 24.8 years & 28.1 years & $1.03 \%$ \\
\hline
\end{tabular}

Fig. 15.10 Canonicalized Table for People.

\begin{tabular}{|l|l|c|}
\hline Country & Location Description & Geographic Coordinates \\
\hline Afghanistan & $\begin{array}{l}\text { Southern Asia, north and west } \\
\text { of Pakistan, east of Iran } \\
\text { Southeastern Europe, bordering } \\
\text { on the Adriatic Sea and Ionian } \\
\text { Sea, between Greece and Serbia } \\
\text { and Montenegro }\end{array}$ & $3300 \mathrm{~N}, 6500 \mathrm{E}$ \\
$\ldots$ & \\
\hline
\end{tabular}

Fig. 15.11 Canonicalized Table for Geography.

tools that let a user efficiently mark a table's label areas, data areas, title, and other augmentations [JN08, $\left.\mathrm{PJK}^{+} 09\right]$. As a principle, it should always be possible for a knowledge worker to manually specify any output to be generated by the system, even though we aim to automate as much as is possible. Manual specification ensures that we can always complete a task and that we can correct any errors our automated procedures may introduce.

\subsubsection{Construction of Mini-Ontologies}

Figure 15.15 gives a graphical representation of each of the mini-ontologies for our six sample canonicalized tables in Figures 15.9-15.14. The notation differs slightly from our earlier notation for OSM-O. In this notation, we represent graphically the four common participation constraints: $0:^{*}, 0: 1$, $1:^{*}$, and 1:1. A zero minimum makes participation optional, which we denote with an "o" on the relationship-set line near the object set whose objects participate optionally. The absence of an "o" makes the participation 


\begin{tabular}{|l|r|}
\hline & \multicolumn{1}{|c|}{ Population } \\
\hline Asia & $3,674,000,000$ \\
Africa & $778,000,000$ \\
$\ldots$ & $8,040,000$ \\
New York City, New York & $3,700,000$ \\
Los Angeles, California & \\
$\ldots$ & $12,150,000$ \\
Mumbai, India & $11,960,000$ \\
Buenos Aires, Argentina & \\
$\ldots$ & $1,256,167,701^{*}$ \\
China & $1,017,645,163^{*}$ \\
India & \\
... & \\
*January 15, 2000 &
\end{tabular}

Fig. 15.12 Canonicalized Table for Largest Populations.

\begin{tabular}{|c|c|c|c|c|c|}
\hline Place & Type & Elevation* & USGS Quad & Lat & Lon \\
\hline Bonnie Lake & reservoir & unknown & Seivern & $3372 \mathrm{~N}$ & $8142 \mathrm{~W}$ \\
\hline Bonnie Lake & lake & unknown & |Mirror Lake & $|4071 \mathrm{~N}|$ & $11088 \mathrm{~W}$ \\
\hline New York & town/city & unknown & Jersey City & $4071 \mathrm{~N}$ & $7401 \mathrm{~W}$ \\
\hline New York & town/city & 149 meters & Leagueville & $3217 \mathrm{~N}$ & $9567 \mathrm{~W}$ \\
\hline $\begin{array}{l}\text { New York } \\
\ldots\end{array}$ & mine & unknown & |Heber City & $\left|\begin{array}{lll}40 & 62 & \mathrm{~N}\end{array}\right|$ & $11149 \mathrm{~W}$ \\
\hline
\end{tabular}

Fig. 15.13 Canonicalized Table for US Topographical Maps.

\begin{tabular}{|c|l|c|l|}
\hline Pos & Language & Speakers & Where Spoken (Major) \\
\hline 1 & Mandarin & $885,000,000$ & China, Malaysia, Taiwan \\
2 & Spanish & $332,000,000$ & South America, Central America, Spain \\
3 & English & $322,000,000$ & USA, UK, Australia, Canada, New Zealand \\
$\ldots$ & &
\end{tabular}

Fig. 15.14 Canonicalized Table for Most Spoken Languages.

mandatory - equivalent to a 1-minumum in a participation constraint. Thus, for example, the mini-ontology in Figure 15(e) declares that a Place must have a Name and may, but need not, have an Elevation. A 1-maximum in a participation constraint makes the relationship set functional, which we denote with an arrowhead on the opposite side of a relationship-set line. Thus, for example, in Figure 15(e), a Place has one Name, at most one Elevation, one USGS Quad (the map in which the center of the place appears), and one pair of Geographic Coordinates. The functional (arrowhead) notation, also allows us to express functional dependencies whose left-hand side is composite. Thus, for example, in Figure 15(a) we have the functional dependency 
Country Religion $\rightarrow$ Percent. The notation also provides explicitly for object values with large black dots, which are object sets (one-place predicates) limited to have a single value.

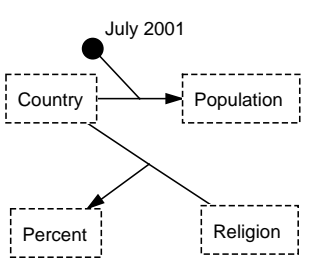

(a)

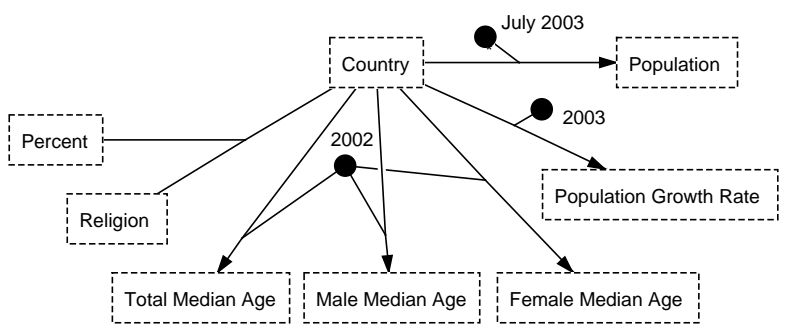

(b)

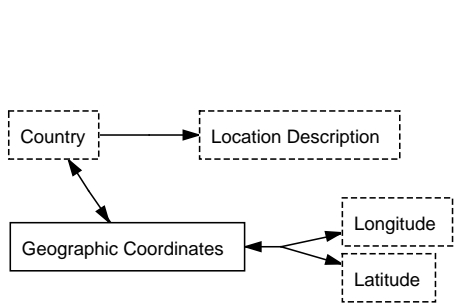

(c)

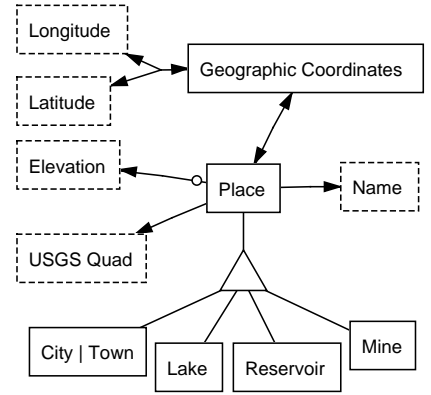

(e)

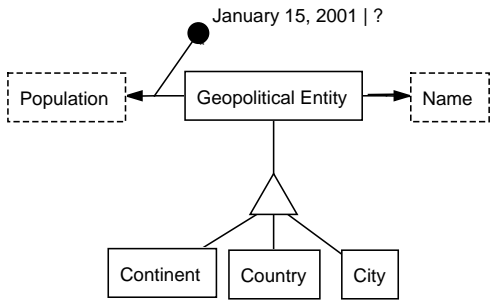

(d)

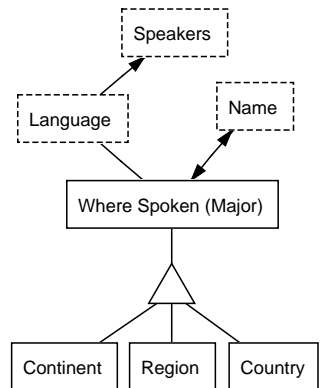

(f)

Fig. 15.15 Mini-Ontologies Constructed from the Tables in Figures 15.9 - 15.14.

To construct mini-ontologies from canonicalized tables, we must discover what concepts (object sets) are involved and how they are related (relationship sets). We must also determine the constraints that hold over the relationship sets (functional, mandatory/optional participation, aggregations) and among the object sets (generalization/specialization). We do so by appealing to the structural constraints of canonicalized tables and to outside resources such as WordNet and a data-frame library [LE09]. Aligning model instances with outside resources also makes them easier to integrate. 
As an example, we obtain the mini-ontology in Figure 15(a) from the table in Figure 15.9 as follows. Country is a key and appears in a leftmost column, strongly suggesting that it should be the tail side of functional dependencies. Population depends on Country but also depends on July 2001. Knowledge from the data frame library recognizes that the values in the Religion columns are Percent values. The religions, which could either be object sets or values, are values since there are many (our current threshold is five). Given that religions are values, we therefore have a ternary relationship among Country, Religion, and Percent. Based on constraint mining, we can determine that Country and Religion together functionally determine Percent. Creation of the remaining five mini-ontologies is similar.

\subsubsection{Mapping Discovery and Ontology Merge}

Our approach to discovering inter-ontology mappings is multi-faceted [EJX01, EJX02], which means that we use all evidence at our disposal to determine how to match concepts. These facets include label matching [EJX01], value similarity [EJX01], expected values via matching values with data frames [EJX01, EXD04], constraints [BE03], and structure [ETL02, EJX02]. In using this evidence we look not only for direct matches as is common in most schema matching techniques [BMPQ04, FNS07, RB01] but also for indirect matches [XE06]. Thus, for example, we are able to split or join columns to match the single Geographic Coordinates column in Figure 15.11 with the pair of columns, Lat and Lon, in Figure 15.13, and we are able to divide the values in the Place column in Figure 15.13 into several different object sets.

Once we have discovered mappings between mini-ontologies or between a mini-ontology and the ontology we are building, we can begin the merge process. Sometimes the match is such that we can directly fuse two ontologies by simply keeping all the nodes and edges of both and merging nodes and edges that directly correspond. Often, however, merging induces conflicts that must be resolved. We resolve conflicts synergistically based on Issue/Default/Suggestion (IDS) statements [BE03, Lia08] When a conflict arises, the system brings the issue to the attention of a knowledge worker. It provides a default resolution - the one it will take if the user does not intervene, and it makes some suggestions about alternate possibilities. In the tool we have created [Lia08], a user can specify mappings that the automated matching algorithms my miss, can remove mappings that the matching algorithms may have incorrectly suggested, can run the merge automatically (allowing the system to take all the default resolutions for any conflict), can run merge interactively (resolving each IDS statement manually), and can manually adjust the results after merge.

Given a collection of mini-ontologies, such as those in Figure 15.15, we look initially for mini-ontologies that exhibit as large of an overlap as possible (as measured by the number of inter-ontology mappings); thereafter we select 
mini-ontologies with the largest overlap with our growing ontology. In our example we begin by merging the mini-ontologies in Figures 15(a) and 15(b).

1st Merge Country matches Country and Population matches Population. Both July 2001 and July 2003 are date components associated with Population, and we merge them as Date.

2nd Merge Building on the 1st Merge, we add the mini-ontology in Figure $15(\mathrm{~d})$ and obtain the emerging ontology in Figure 15.16. Here, we encounter IDS statements that help us reconcile the lexical/non-lexical Country object sets so that Country becomes non-lexical with an associated name and also that let Population be an inherited property and thus omit it from the Country specialization.

3rd Merge Continuing, we merge the mini-ontology in Figure 15(f) with the growing ontology in Figure 15.16. Here, the data in the object sets Geopolitical Entity and Where Spoken largely overlap, but it is not 100\% clear whether one set should be a subset of the other, whether they are overlapping siblings in an is-a hierarchy, or whether they should be the same set. An IDS statement is therefore appropriate, and we assume the issue is resolved by declaring that the sets are the same and should be called Geopolitical Entity. This merge thus adds Region as a specialization of Geopolitical Entity and adds Language and Speakers connected to Geopolitical Entity in the same way they are connected to Where Spoken (Major) in Figure 15(f).

4th Merge Continuing, we next add the mini-ontology in Figure 15(c). Here, the constraints on the Location Description in Figure 15(c) declare that the relationship is mandatory for both Country and Location Description and functional from Country to Location Description. Because of the lack of location descriptions for most countries in our growing collection, however, we have enough evidence to override the mandatory declaration and make the relationship for Country optional.

5th Merge Continuing, we next add the mini-ontology in Figure 15(e) and obtain the growing ontology in Figure 15.17. Here, with the help of IDS statements, we must recognize that Geopolitical Entity is a specialization of Place. Other adjustments come readily, including inheriting Name from Place and making the existence of USGS Quad optional for Place based on lack of map locations for most places.

As we transform tables to mini-ontologies and merge them, we also retain the data. The end result is a populated ontology and thus a KB that represents the domain described by the given collection of tables. 


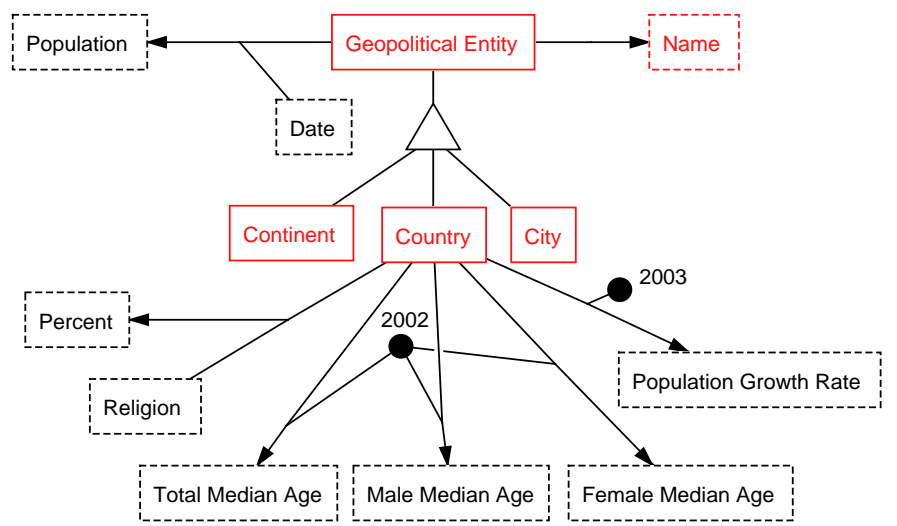

Fig. 15.16 Growing Ontology after Merging the Mini-Ontologies in Figures 15(a), 15(b), and $15(\mathrm{~d})$. (The object sets with lighter-shaded, "red," borders are those most recently added.)

\subsubsection{Construction via Form Filling}

Although the KB construction methods discussed in Subsections 15.4.115.4.3 are largely automatic, they have the disadvantage that users have little or no control over the ontological structure created and the data that populates the ontological structure. Users could take the final generated result and edit it by hand - a reasonable possibility if the desired ontological structure and the data are almost the same as the generated structure and data. In this subsection, we discuss an alternative that gives users a way to create a custom-designed ontological structure for a $\mathrm{KB}$ and to populate it with values harvested from a diverse collection of web pages. This method works particularly well when the information to be collected for the KB comes from machine-generated collections of semi-structured web pages such as those commonly found in most hidden-web/deep-web sites.

FOCIH (Forms-based Ontology Creation and Information Harvesting) [TEL09] is a tool that lets users specify ontologies without having to know any conceptual-modeling language or any ontology language. We observe that forms are a natural way for humans to collect information. As an everyday activity, people create forms and ask others to fill in the blanks. FOCIH lets users create their own forms to describe information they wish to harvest. Once defined, users can fill in forms from web pages by copy and paste. From the form specification and user cut-and-paste actions, FOCIH generates an ontology, extracts data, and annotates the web page with respect to the ontology. Further, if the web page is machine-generated and has sibling pages, FOCIH is able to harvest the specified information from all the sibling pages, often without further user intervention. 


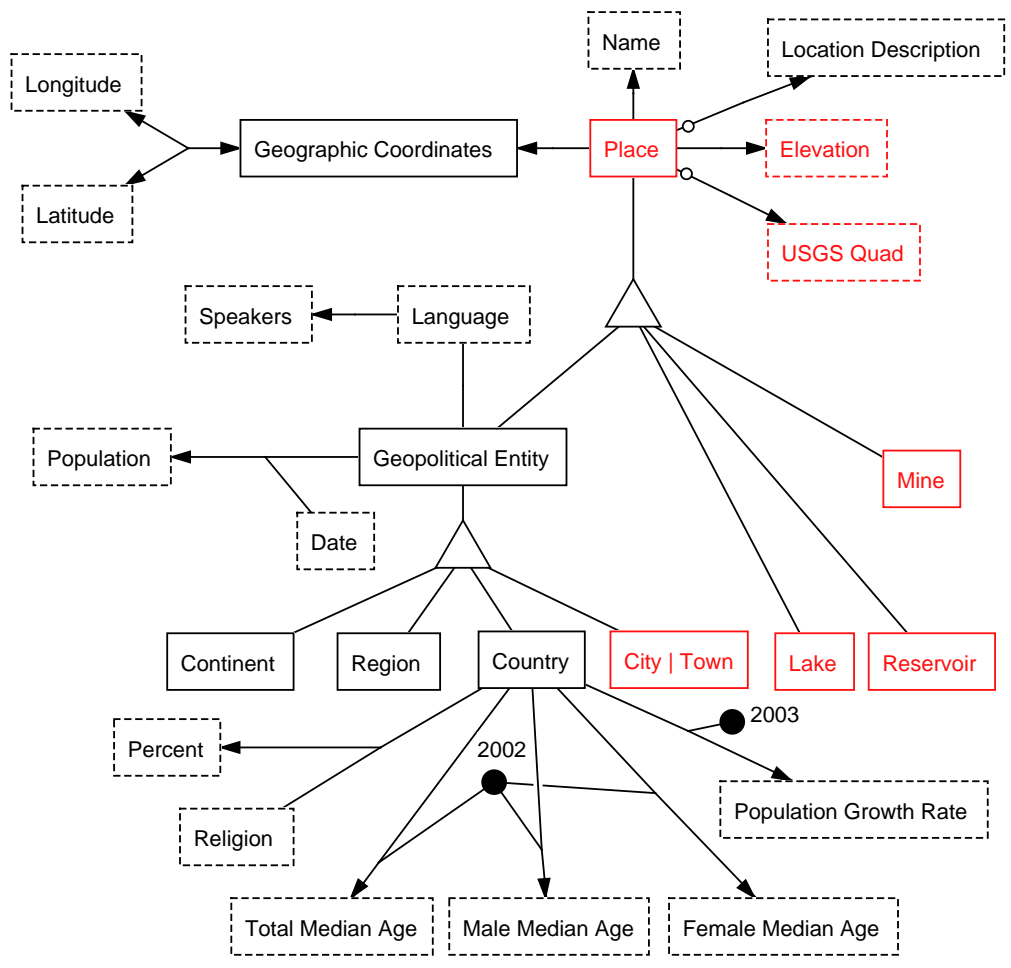

Fig. 15.17 Growing Ontology after Merging all Mini-Ontologies. (The object sets with lighter-shaded, "red," borders are those most recently added.)

FOCIH's form-creation mode provides users with an intuitive method for defining different kinds of form features. FOCIH has five types of form fields: single-label/single-value, single-label/multiple-value, multiple-label/multiplevalue, mutually-exclusive choice, and non-exclusive choice. Users create standard forms by stringing these form elements together in any order and nesting them within one another to any depth. The form in the left panel of Figure 15.18 shows an example. The form is for collecting country information. It starts with three single-label/single-value fields for Name, Capital, and Geographic Coordinates, followed by a single-label/multiple-value field for Religion, and a multiple-label/multiple-value field for Population-Year estimates. The Life Expectancy field is a non-exclusive choice field for either Male or Female Life Expectancy or both. The final field shows the nesting of three form fields for Water, Land, and Total under a single-label/single-value field for Area.

FOCIH's form-fill-in mode lets users browse to a web page they wish to annotate and copy and paste values into form fields. A user highlights values in the page and then clicks on the form field to fill in a value. Figure 15.18 shows a web page for the Czech Republic in the right panel. Copied values 


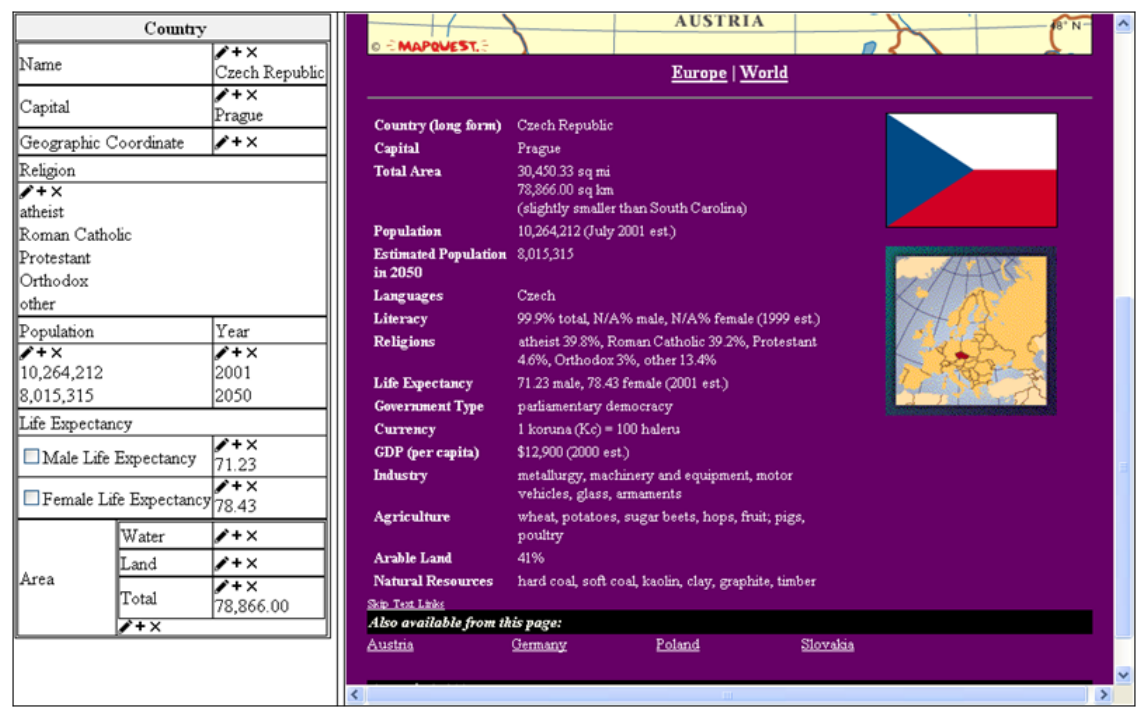

Fig. 15.18 Filled-in FOCIH Form.

from the web page appear in the form in the left panel. The pencil icon lets a user drop a highlighted value into a form field, and the $\mathrm{x}$ icon lets a user remove a value. The plus icon lets a user concatenate a second part of the value to a partial value already in the form field. Thus, for example, if the latitude and longitude values are disjoint, perhaps labeled Latitude: and Longitude: and appearing on separate lines in a web page, a user can concatenate the two as a single value in the Geographic Coordinates field.

From the filled-in form, FOCIH can generate a conceptual model and populate it with values. Note that filled-in nested forms are identical in structure to the nested tables discussed in Subsection 15.4.2. Thus, the generated ontologies are similar, and are like the OSM-O model instance in Figure 15.7. In addition to generating a conceptual model and populating it, FOCIH also records the following information: (1) paths to leaf nodes in the DOM tree of an HTML page containing each value and, for concatenated values, each value component; (2) for each value the most specific instance recognizer from the data-frame library (e.g., string, number, percentage, year, geographic coordinate); and (3) enough left, right, and delimiter context within each leaf node to identify the value or values within the DOM-tree node. This enables FO$\mathrm{CIH}$ to harvest the same information from other machine-generated sibling pages from the same web site.

The result of running FOCIH over a collection of sibling pages is a custombuilt KB containing the information in the collection. Further, FOCIH can harvest information from other sibling-page collections with respect to the same custom-built ontology, which can further augment the KB. 


\subsection{WoK Usage}

The construction of extraction ontologies leads to "understanding" within a WoK. This "understanding" leads to the ability to answer a free-form query because, as we explain in this section, a WoK system can identify an extraction ontology that applies to a query and match the query to the ontology. Hence, a WoK system can reformulate the free-form query as a formal query, so that it can be executed over a KB. In addition, "understanding" leads to establishing a context of discourse, allowing the system to expose its conceptualization of the subject and thus allowing users to more effectively communicate their information needs to the system. In both cases results returned for a query include not only answers to queries but also answer justification. Users can obtain a reasoning chain justifying each answer as well as provenance links identifying each ground fact supporting the answer.

Definition 11. Let $S$ be a source conceptualization and let $T$ be a target conceptualization formalized as an OSM-EO model instance. We say that $T$ understands $S$ if there exists an $S$-to- $T$ transformation that maps each one-place predicate of $S$ to an object set of $T$, each $n$-place predicate of $S$ to an $n$-place relationship set of $T(n \geq 2)$, each fact of $S$ to a fact of $T$ with respect to the predicate mappings, and each operator of $S$ to an operator in a data frame of $T$, such that the constraints of $T$ all hold over the transformed predicates and facts.

Observe that although Definition 11 states how $T$ is formalized, it does not state how $S$ is formalized. Thus, the predicates and operators of $S$ may or may not be directly specified. This is the hard part of "understanding"-to recognize the applicable predicates and operators. But this is exactly what extraction ontologies are meant to do. If an OSM-EO model instance is linguistically well grounded, then it can "understand" so long as what is stated in $S$ is within the context of $T$ - that is if there is an object set or relationship set in $T$ for every predicate in $S$ and if there is an operator in a data frame of $T$ for every operator in $S$.

Applications of understanding include free-form query processing, grounded reasoning chains, and $\mathrm{KB}$ building for research studies. We explain and illustrate each in turn. In doing so, we also illustrate our WoK prototype system, which we are building as a way to experiment with our vision of a WoK $\left[\mathrm{ELL}^{+} 08\right]$.

\subsubsection{Free-Form Query Processing}

Figure 15.19 illustrates free-form query processing within our WoK prototype. To "understand" a user query, our WoK prototype first determines which extraction ontology applies to the query by seeing which one recognizes the 


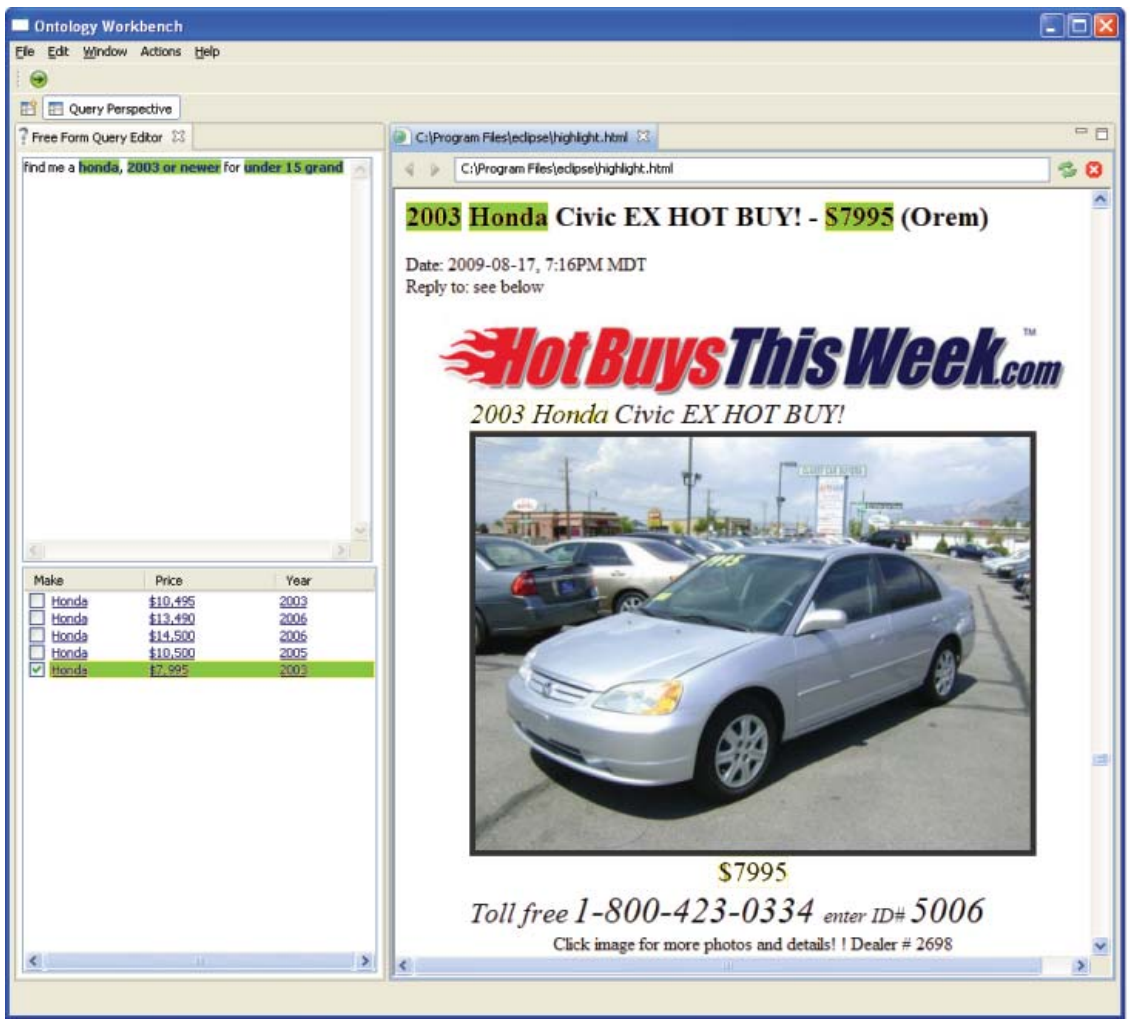

Fig. 15.19 Screenshot of WoK Prototype Showing Free-Form Query Processing.

most instances, predicates, and operators in the query request. For the query in Figure 15.19, we assume that the WoK prototype chooses the Car extraction ontology illustrated in Figures 15.2 and 15.3. The WoK prototype then applies the $S$-to- $T$ transformation highlighting what it "understands" ("Find me a honda, 2003 or newer for under 15 grand "). Figure 15.20 shows the result of this transformation - each predicate and each operation is mapped correctly and the constraints of the OSM-EO model instance all hold. Given this "understanding," it is straightforward to generate a SPARQL query. Before executing the query, our WoK prototype augments it so that it also obtains the stored annotation links. Then, when our WoK prototype displays the results of the query (e.g., in the lower-left box in Figure 15.19), it makes returned values clickable. Clicking on a value, causes our WoK prototype to find the page from which the value was extracted, highlight it, and display the page appropriately scrolled to the location that includes the value. The right panel of Figure 15.19 shows several highlighted values, which happens when a user checks one or more check-boxes before clicking.

The form in Figure 15.20 is for an alerter system which we have implemented for craigslist.org. We use the form in two ways: (1) for comprehensive 


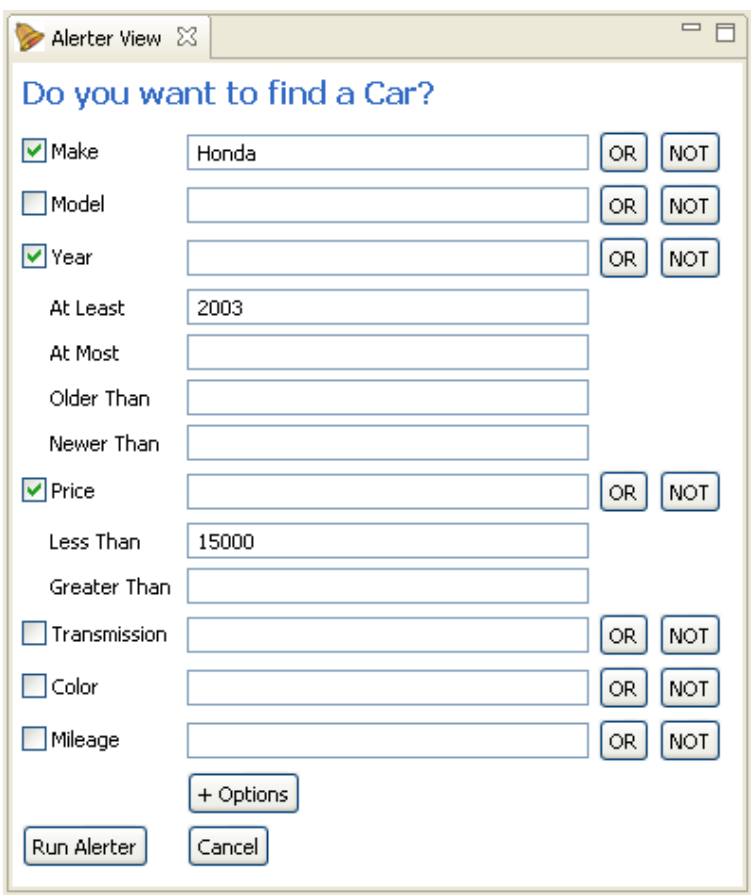

Fig. 15.20 Generated Form Showing the System's "Understanding"-Its "Understood" Instances within Its "Understood" Ontological Context.

feedback to indicate its "understanding" of the query and (2) for giving users advanced options for query specification. As feedback, it lets users know the context in which the system "understands" the query being asked (i.e., the system displays the name of the extraction ontology and its details as form elements), and (2) it lets users know exactly what has been "understood" (i.e., the system displays constant values in fields for object sets or for operations applicable to object sets). With respect to advanced options, it lets users know what else can be asked in the context of the query. A user then has the opportunity to adjust the query or add additional constraints. For example, besides Hondas, a user may wish to also know if Toyotas are for sale but only if they if they are not Camrys. Clicking on $O R$ for Make and adding Toyota and then clicking on NOT for Model and adding Camry makes this possible. The plus icons show that more operators are available; clicking on the plus displays them. For example, a user may wish to limit the search to cars whose odometer reading is less than $100 \mathrm{~K}$ miles; clicking on the "+ Options" button shows the Boolean operators for Mileage and lets a user enter this limitation. 


\subsubsection{Grounded Reasoning Chains}

To illustrate grounded reasoning chains, we give an example from familyhistory research. Many millions of hand-written records such as those in the census record in Figure 15.21 have been transcribed by human indexers [Fam]. Using extraction ontologies, we can extract from the transcription to associate names, dates, places, and other information with a genealogical ontology. Bounding boxes for names and other information in the image are also available, so we know where in the image information appears.

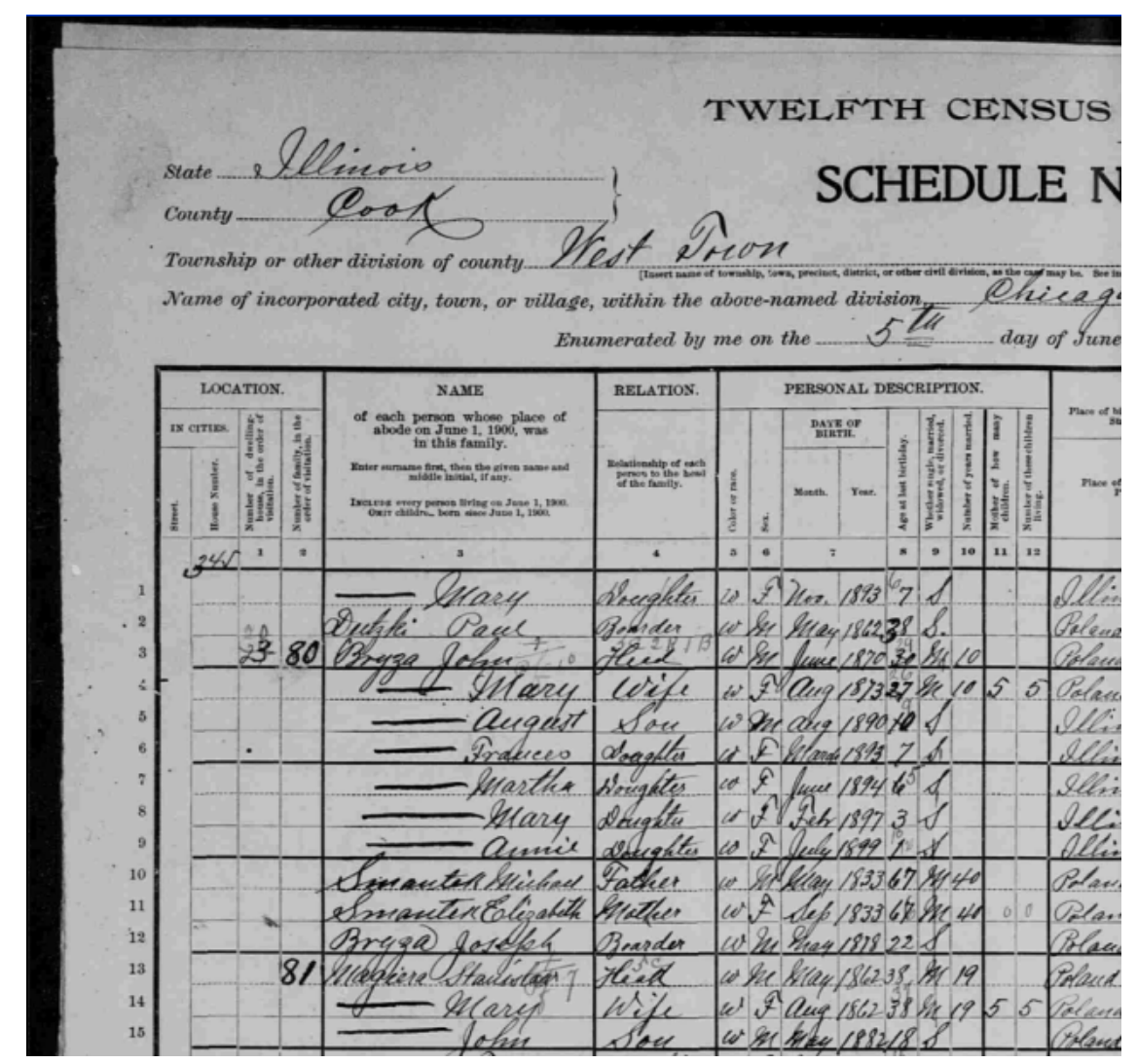

Fig. 15.21 Census Record.

It is not hard to see that, among others, the following rules hold and are useful for establishing family relationships implied by the information in the census record in Figure 15.21:

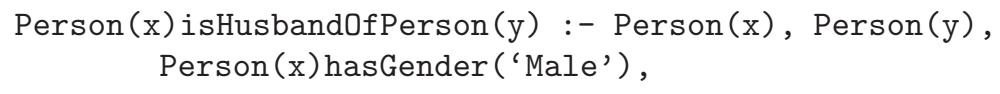




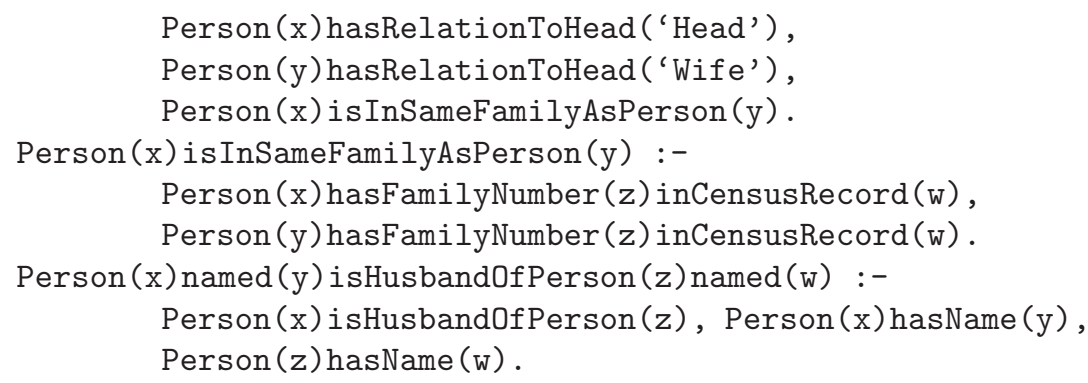

The first rule states that a person $x$ is the husband of person $y$ if $x$ is a male head of the family, $y$ is the wife, and $x$ and $y$ are in the same family. The second rule assures that they are in the same family by checking to see that their family number is the same, and the last rule associates the husband and wife with their names.

When we associate a rule with an ontology, we must ensure that it is grounded in the base predicates of the ontology. The set of rules forms a graph over a "head predicate depends-on body predicate" relation, and this graph must lead to predicates declared in the ontology as object-set predicates, relationship-set predicates or Boolean operations declared for the ontology. Recursive rules such as the following rules to compute ancestors are possible, but must also be grounded.

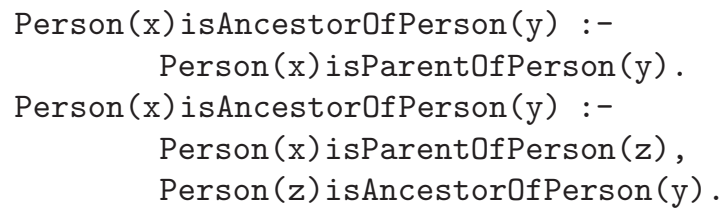

Here, the predicate Person(x)isParentOfPerson(y) must be a rule head that eventually resolves down to ground predicates such as Person $(x)$ hasRelationToHead('Son') or Person(x)hasRelationToHead('Daughter').

We linguistically ground a rule $r$ by declaring a data frame for $r$ in the same way we declare a data frame for an object set (if the head of $r$ is a one-place predicate) or for a relationship set (if the head of $r$ is an $n$-place predicates, $n \geq 2)$. Thus, for example, $\{$ Person $\} \backslash s^{*} i s \backslash .{ }^{*}$ husband $\backslash s^{*}$ of $\backslash s^{*}\{$ Person $\}$ may be one of the regular expressions for the rule head Person $(x)$ isHusbandOf$\operatorname{Person}(y)$.

Now when we ask the query, "Who is the husband of Mary Bryza?", we can match the query to our genealogy ontology and specifically to the rule and thus also the chain of rules needed to answer the query. The returned result would yield "John Bryza" and perhaps others if other Mary Bryzas are known within the KB. Clicking on "John Bryza" yields both the reasoning chain in Figure 15.22 and the highlighted census recored in Figure 15.23. The reasoning chain is simply a list of rules invoked with instance data filled in for the variables and reformatted to be more readable. The highlighted census 
record shows the source of all the extracted ground fact values used to yield the answer.

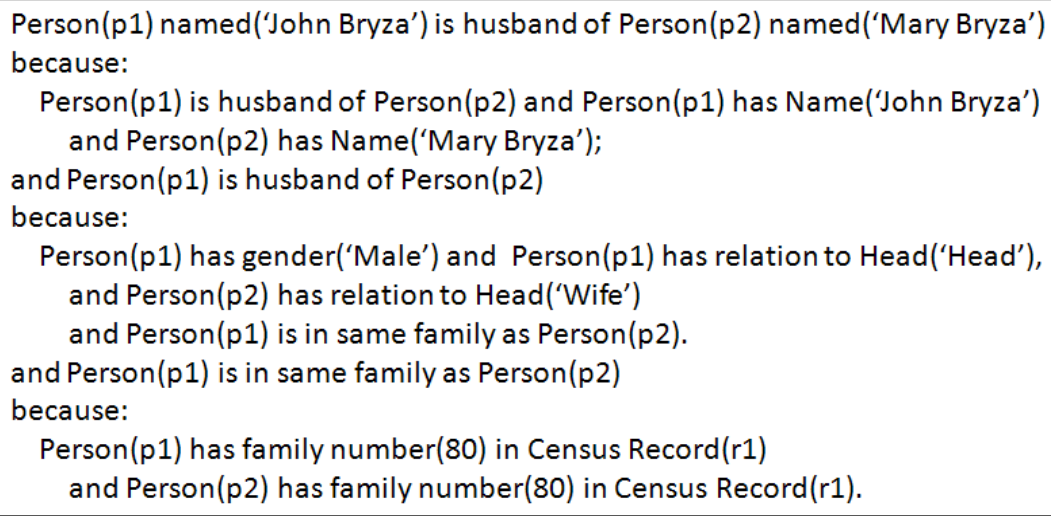

Fig. 15.22 Reasoning Chain for Query.

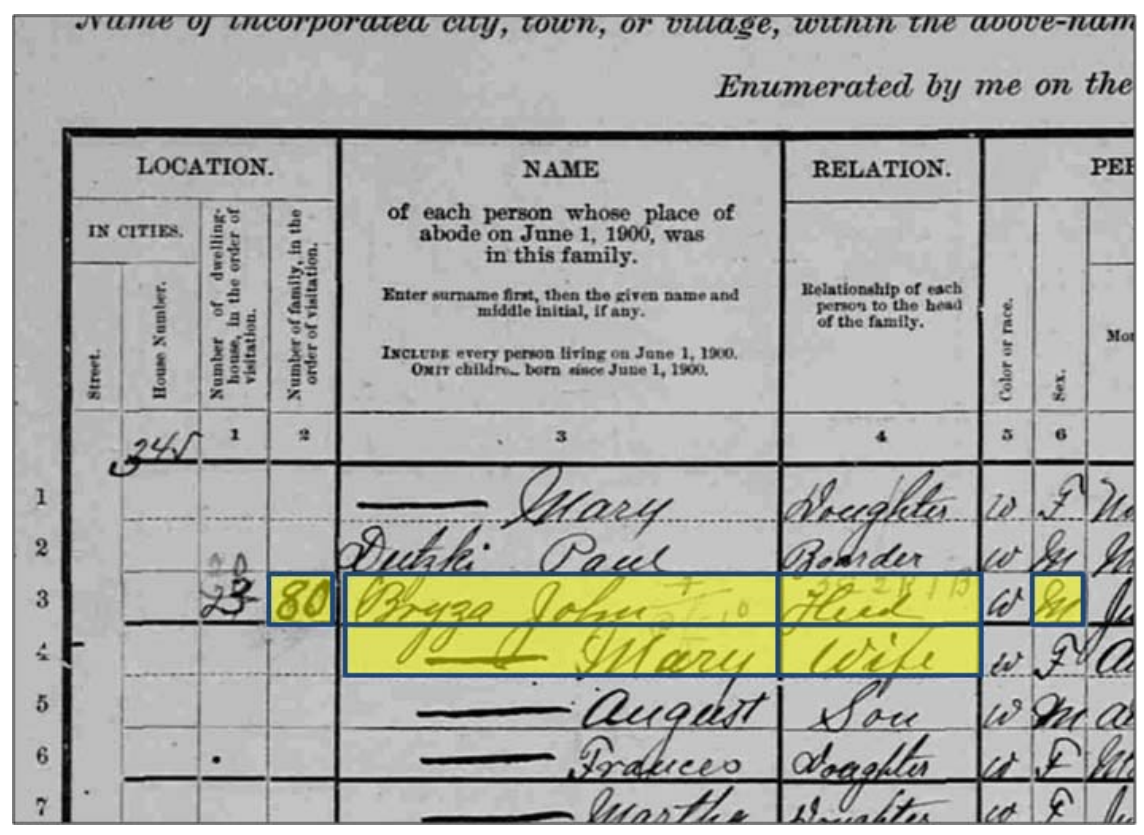

Fig. 15.23 Census Record with Ground Facts Highlighted. 


\subsubsection{Knowledge Bundles for Research Studies}

In addition to "understanding" queries, it should be clear that "understanding" is also about fact finding. The fundamental intent of linguistically grounding extraction ontologies is to allow them to recognize facts in structured, semi-structured, and unstructured text. As an example, we give a plausible scenario, based on the WoK components we have presented, for gathering facts for a bio-research study and storing them as a KB for further analysis $\left[\mathrm{ELL}^{+} 09\right]$. Gathering tasks for these research studies often take trained bio-researchers several man-months of work. So, any significant speedup extraction ontologies can provide would be of great benefit in bio-medical research.

Suppose a bio-researcher $B$ wishes to study the association of TP53 polymorphism and lung-cancer. To do this study, $B$ wants information from the NCBI dbSNP repository ${ }^{3}$ about SNPs (chromosome location, SNP ID and build, gene location, codon, and protein), about alleles (amino acids and nucleotides), and about the nomenclature for amino-acid levels and nucleotide levels. $B$ also needs data about human subjects with lung cancer and needs to relate the SNP information to human-subject information.

To gather information from dbSNP, $B$ uses FOCIH to construct the form in the left panel in Figure 15.24. Form construction consists of selecting types of form fields and organizing and nesting form fields so that they are a conceptualization of the information $B$ wishes to harvest for the research study. $B$ next finds a first SNP page in dbSNP from which to begin harvesting information. (The created form and located page need not have any special correspondence - no schema correspondence, no name correspondence, and no special structure requirements - but, of course, the page should have data of interest for the research study and thus for the created form.) $B$ then fills in the form by cut-and-paste actions, copying data from the page in the center panel in Figure 15.24 to the form in the left panel.

To harvest similar information from the numerous other dbSNP pages, $B$ gives a list of URLs, as the right panel in Figure 15.24 illustrates (although there would likely be hundreds rather than just the six in Figure 15.24). The FOCIH system automatically harvests the desired information from the dbSNP pages referenced in the URL list. Since one of the challenges bioresearchers face is searching through the pages to determine which ones contain the desired information, FOCIH should provide a filtering mechanism. By adding constraints to form fields, bio-researchers can cause the FOCIH harvester to gather information only from pages that satisfy the constraints. $B$, for example, might only want coding SNP data with a significant heterogeneity (i.e., minor allele frequency $>1 \%$ ).

\footnotetext{
3 The Single Nucleotide Polymorphism database (dbSNP) is a public-domain archive for a broad collection of simple genetic polymorphisms hosted by National Center for Biotechnology Information (NCBI) at www.ncbi.nlm.nih.gov/projects/SNP/.
} 


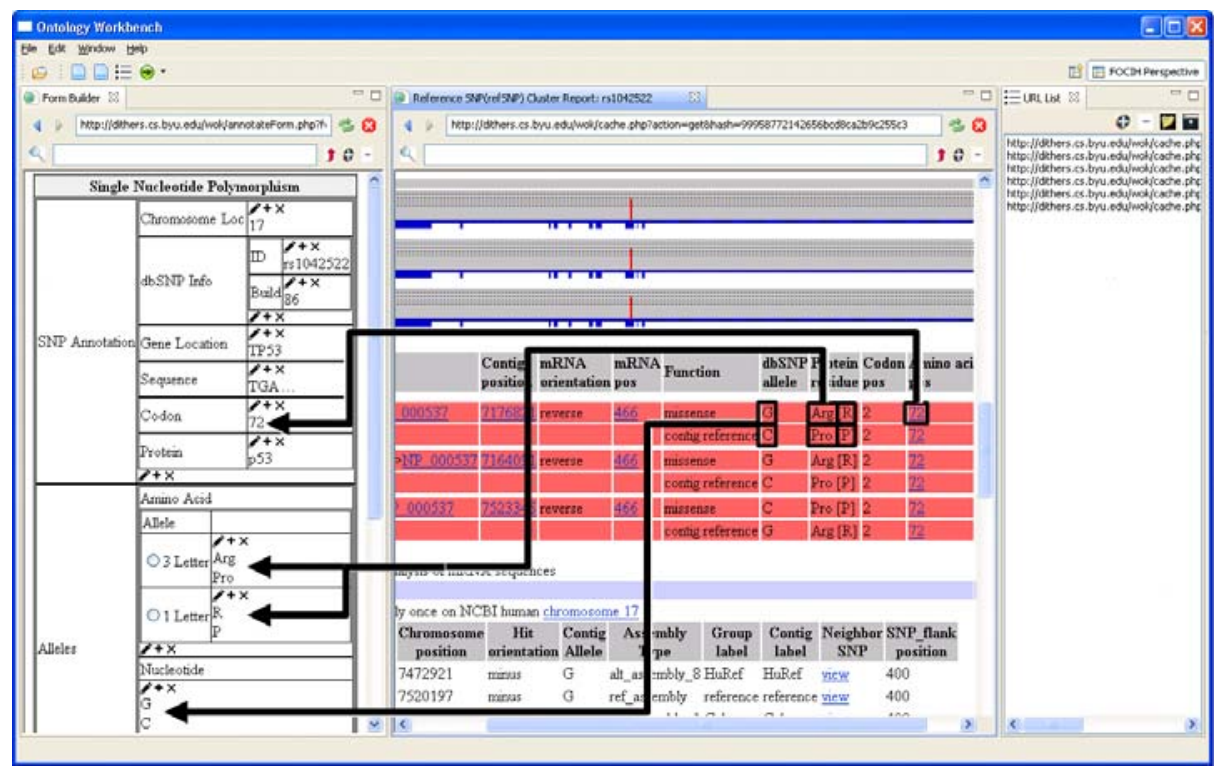

Fig. 15.24 Form Filled in with Information from an SNP Page.

For the research scenario, $B$ may also wish to harvest information from other sites such as GeneCard. $B$ can use FOCIH with the same form to harvest from as many sites as desired. Interestingly, however, once FOCIH harvests from one site, it can use the knowledge it has already gathered to do some of the initial cut-and-paste for $B$. In addition to just being a structured knowledge repository, the $\mathrm{KB}$ being produced also becomes an extraction ontology capable of recognizing data items it has already seen. It can also recognize data items it has not seen but are like the data it has seen - e.g., numeric values or DNA snippets.

Using KBs as extraction ontologies also lets bio-researchers search the literature. Suppose $B$ wishes to find papers related to the information harvested from the dbSNP pages. $B$ can point the extraction ontology to a repository of papers to search and cull out those that are relevant to the study. Using the $\mathrm{KB}$ as an extraction ontology provides a sophisticated query of the type used in information retrieval resulting in high-precision document filtering. For example, the extraction ontology recognizes the highlighted words and phrases in the portion of the paper in Figure 15.25. With the high density of not only keywords but also data values and relationships all aligned with the ontological KB, the system can designate this paper as being relevant for $B$ 's study.

For collecting human-subject information, $B$ may decide to obtain information from InDivo, a database containing personally controlled health records. Based on reverse-engineering techniques, the system can automat- 


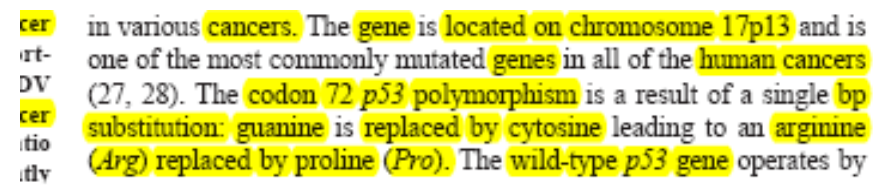

Fig. 15.25 Paper Retrieved from PMID Using an Extraction Ontology.

\begin{tabular}{|c|c|c|c|}
\hline \multicolumn{4}{|c|}{ Medical Document } \\
\hline \multirow{6}{*}{ Demographics } & \multicolumn{2}{|l|}{ Sex } & \\
\hline & \multicolumn{2}{|l|}{ DateofBirth } & \\
\hline & \multicolumn{2}{|l|}{ Deceased } & \\
\hline & \multicolumn{2}{|l|}{ Race } & \\
\hline & \multicolumn{2}{|l|}{ Ethnicity } & \\
\hline & \multicolumn{2}{|c|}{ HighestEducationLevel } & \\
\hline \multirow{3}{*}{ Family History } & Condition & Relationship & \\
\hline & & & \\
\hline & Prescription & Dose & Duration \\
\hline Medication & & & \\
\hline
\end{tabular}

Fig. 15.26 Some Human Subject Information Reverse-Engineered from INDIVO.

ically reverse-engineer the INDIVO database to a $\mathrm{KB}$, and present $B$ with a form representing the schema of the database. Figure 15.26 shows an example. $B$ can then modify the form, deleting fields not of interest and rearranging fields to suit the needs of the study. Further, $B$ can add constraints to the fields so that the the systems only gathers data of interest.

With all information harvested and organized into a $\mathrm{KB}, B$ can now issue queries and reason about the data to do some interesting analysis. Figure 15.27 shows a sample SPARQL query over the data harvested from the pages referenced by the six URLs listed in Figure 15.24. The query finds three SNPs that satisfy the query's criteria and for each, returns the dbSNP ID, the gene location, and the protein residue. As Figure 15.27 shows, $B$ wishes to see the source of the query result $<$ rs55819519, TP53, His Arg $>$. 


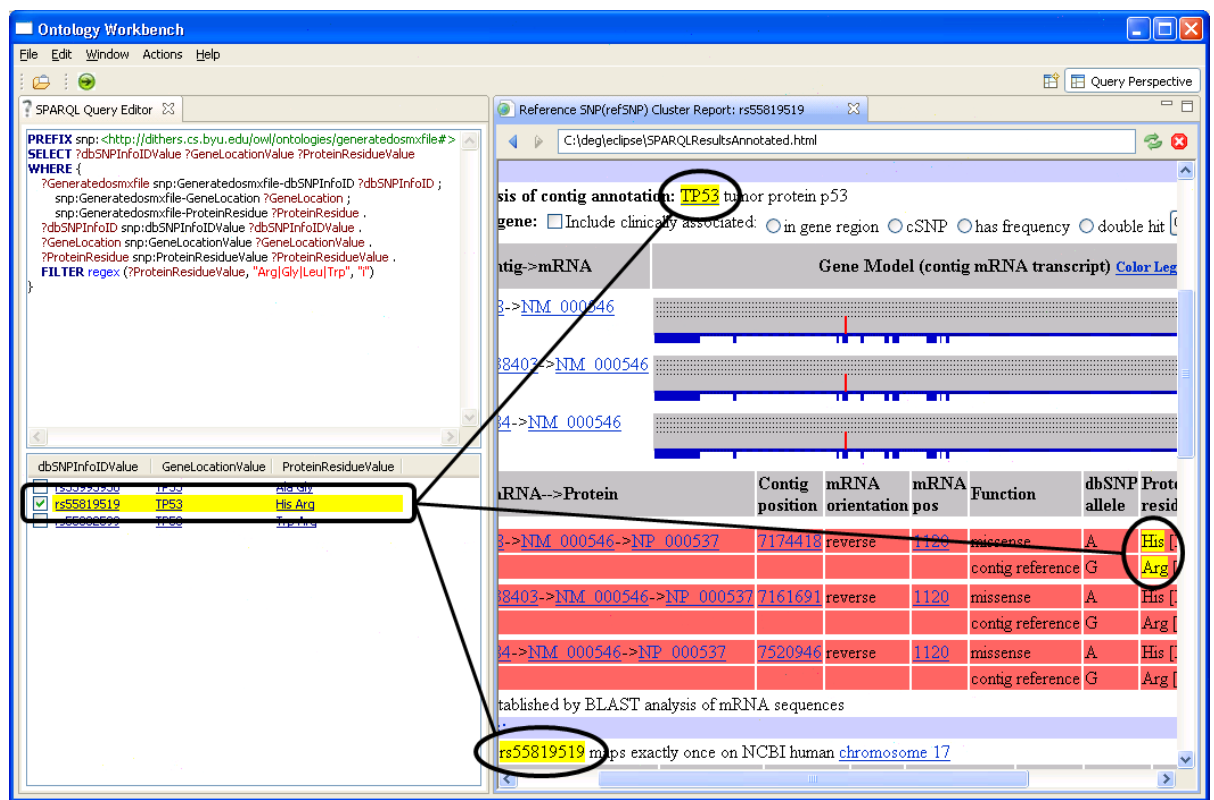

Fig. 15.27 Screenshot of our Web of Knowledge Prototype.

\subsection{Conclusion}

We have described a web of knowledge (WoK) as a collection of interconnected KBs superimposed over a web of documents. Our WoK vision has conceptual modeling at its foundation. As described, a WoK consists of KBs, which are conceptual-model instances augmented with facilities that provide (1) for both extensional and intensional facts, (2) for linking between KBs yielding a web of data, and (3) for authentication by linking to source documents and explicating reasoning chains.

We have provided a formal foundation for WoK components - ontologies (OSM-O) in terms of decidable first-order logic and extraction ontologies (OSM-EO) linguistically grounded via data-frame recognizers. In addition, we have formalized a WoK as a collection of interconnected knowledge bundles (KBs) consisting of OSM-EO model instances with valid interpretations super-imposed over source documents.

Further, we have addressed concerns about WoK construction. Transformations map source conceptualizations to target conceptualizations. Information- and constraint-preserving transformations guarantee that target conceptualizations completely and accurately capture source conceptualizations. We have explained how reverse engineering of some documents can yield source conceptualizations guaranteed to preserve information and constraints. We conclude, however, that many source conceptualizations (rang- 
ing from semi-structured sources such as ordinary human-readable tables and forms to unstructured sources such as free-running text) likely require besteffort automation methods and may involve some user supervision. We have given, as examples, a way to transform a collection of ordinary tables with overlapping information from some domain into a $\mathrm{KB}$ and a way to construct KBs via form-creation and form-filling.

Finally, we have addressed concerns about WoK usage. When transformations exist that map source predicates and operations to an established ontology, the ontology is said to have "understood" the information in the source. "Understanding" applied to free-form queries allows untrained users to query the envisioned WoK. Users receive direct answers to queries, rather than pages that may contain answers. They may, however, ask for justification by clicking on displayed answers, which yields the pages from which the answers were taken and also yields an explanation of any reasoning used to generate inferred answers for the query. As another example of WoK usage, we have illustrated the process of creating a KB for bio-medical research studies.

We have implemented a WoK prototype $\left[\mathrm{ELL}^{+} 08\right]$ including some prototypical extraction ontologies [ECJ $\left.{ }^{+} 99\right]$. We have also done some work on automated extraction-ontology construction [TE09, LE09, TEL09, TEL ${ }^{+} 05$ ] and some work on free-form query processing [Vic06, AME07]. We nevertheless still have much work to do, even on fundamental WoK components such as creating a sharable data-frame library, constructing data frames for relationship sets, finding ways to more easily produce instance recognizers, developing processes for reverse-engineering additional genres of semi-structured sources into KBs, investigating bootstrapping as a way to construct extraction ontologies, enhancing query processing, incorporating reasoning, and addressing performance scalability. We also see many opportunities for incorporating the vast amount of work done by others on information extraction, information integration, and record linkage. We cite as relevant examples: KnowItAll [ECD ${ }^{+}$05], OMNIVORE [Caf09], best-effort information extraction [SDM ${ }^{+}$08], C-PANKOW [CLS05], Q/A systems [RFRF08], bootstrapping pay-as-you-go data integration [SDH08], large-scale deduplication [ARS09], and OpenDMAP [HLF $\left.{ }^{+} 08\right]$.

These collective efforts will eventually lead to a WoK - a realization of ideas of visionaries from Bush [Bus45] to Berners-Lee [BLHL01] and Weikum [WKRS09]. Conceptual modeling can and should play a foundational role in these efforts.

Acknowledgements: Many students and colleagues have contributed in various ways to the web-of-knowledge vision presented here - as colleagues: Douglas M. Campbell, George Nagy, Dennis Ng, Dallan Quass, Yuri Tijerino; as PhD students: Reema Al-Kamha, Muhammed Al-Muhammed, Yihong Ding, Aaron Stewart, Cui Tao, Li Xu; as MS students: Tim Chartrand, Wendy Chen, Xueqi Helen Chen, Kimball Hewett, David Jackman, Stephen Jiang, Zonghui Lian, Stephen Lynn, Rob Lyon, Lars Olson, Craig Parker, 
Clint Tustison, Randy Smith, Mark Vickers, Troy Walker, Alan Wessman, Charla Woodbury, Sai Ho Tony Yau, Yuanqiu Joe Zhou, Andrew Zitzelberger; and as undergraduate research assistants: Robert Clawson, Derek McDaniel, Jeff Peters, Robby Watts. We appreciate the support of the National Science Foundation, whose grants have assisted many of these students in their educational endeavors (Grant Numbers: IIS0083127 \& IIS0414644).

\section{References}

[AH08] D. Allemang and J. Hendler. Semantic Web for the Working Ontologist: Effective Modeling in RDFS and OWL. Morgan Kaufmann Publishers, Burlington, Massachusetts, 2008.

[Aik98] P.H. Aiken. Reverse engineering of data. IBM Systems Journal, 37(2):246-269, 1998.

[AK07] R. Al-Kamha. Conceptual XML for Systems Analysis. PhD dissertation, Brigham Young University, Department of Computer Science, June 2007.

[AME07] M. Al-Muhammed and D.W. Embley. Ontology-based constraint recognition for free-form service requests. In Proceedings of the 23rd International Conference on Data Engineering (ICDE'07), pages 366-375, Istanbul, Turkey, April 2007.

[AriBC] Aristotle. Metaphysics. Oxford University Press, New York, about 350BC. (1993 translation).

[ARS09] A. Arasu, C. Re, and D. Suciu. Large-scale deduplication with constraints using Dedupalog. In Proceedings of the 25th International Conference on Data Engineering (ICDE 2009), pages 952-963, Shanghi, China, March/April 2009.

[BCHS09] P. Buitelaar, P. Cimiano, P. Haase, and M. Sintek. Towards linguistically grounded ontologies. In Proceedings of the 6th European Semantic Web Conference (ESWC'09), pages 111-125, Heraklion, Greece, May/June 2009.

[BE03] J. Biskup and D.W. Embley. Extracting information from heterogeneous information sources using ontologically specified target views. Information Systems, 28(3):169-212, 2003.

[BL99] T Berners-Lee. Weaving the Web. Harper SanFrancisco, San Francisco, California, 1999.

[BLHL01] T. Berners-Lee, J. Hendler, and O. Lassila. The semantic web. Scientific American, 36(25):34-43, May 2001.

[BMPQ04] P.A. Bernstein, S. Melnik, M. Petropoulos, and C. Quix. Industrial-strength schema matching. SIGMOD Record, 33(4):38-43, December 2004.

[BN03] F. Baader and W. Nutt. Basic description logics. In F. Baader, D. Calvanese, D. McGuinness, D. Nardi, and P. Patel-Schneider, editors, The Description Logic Handbook, chapter 2, pages 43-95. Cambridge University Press, Cambridge, UK, 2003.

[BN08] J. Bleiholder and F. Naumann. Data fusion. ACM Computing Surveys, 41(1):1-41, December 2008.

[Bus45] V. Bush. As we may think. The Atlantic Monthly, 176(1):101-108, July 1945.

[Caf09] M.J. Cafarella. Extracting and querying a comprehensive web database. In Proceedings of the Conference on Innovative Data Systems Research (CIDR'09), Monterey, California, 2009.

[CGL09] A. Calì, G. Gottlob, and T. Lukasiewicz. Tractable query answering over ontologies with Datalog ${ }^{ \pm}$. In Proceedings of the DL Home 22nd International 
Workshop on Description Logics (DL 2009), Oxford, United Kingdom, July 2009.

[CGP09] A. Cali, G. Gottlob, and A. Pieris. Tractable query answering over conceptual schemata. In Proceedings of the 28th International Conference on Conceptual Modeling (ER 2009), pages 175-190, Gramado, Brazil, November 2009.

[Cim06] P. Cimiano. Ontology Learning and Population from Text: Algorithms, Evaluation and Applications. Springer Verlag, New York, New York, 2006.

[CLS05] P. Cimiano, G. Ladwig, and S. Staab. Gimme' the context: Context-driven automatic semantic annotation with C-PANKOW. In Proceedings of the 14 th International World Wide Web Conference (WWW205), pages 332-341, Chiba, Japan, May 2005.

[dSJT06] A.C. de Silva, A.M. Jorge, and L. Torgo. Design of an end-to-end method to extract information from tables. International Journal of Document Analysis and Recognition, 8(2):144-171, 2006.

$\left[\mathrm{ECD}^{+}\right.$05] O. Etzioni, M. Cafarella, D. Downey, S. Kok, A. Popescu, T. Shaked, S. Soderland, D. Weld, and A. Yates. Unsupervised named-entity extraction from the web: An experimental study. Artificial Intelligence, 165(1):91-134, 2005.

[ECJ+99] D.W. Embley, D.M. Campbell, Y.S. Jiang, S.W. Liddle, D.W. Lonsdale, Y.-K. $\mathrm{Ng}$, and R.D. Smith. Conceptual-model-based data extraction from multiplerecord web pages. Data 83 Knowledge Engineering, 31(3):227-251, November 1999.

[EHLN06] D.W. Embley, M. Hurst, D. Lopresti, and G. Nagy. Table-processing paradigms: A research survey. International Journal of Document Analysis, 8(2):66-86, 2006.

[EIV07] A.K. Elmagarmid, P.G. Ipeirotis, and V.S. Verykios. Duplicate record detection: A survey. IEEE Transactions on Knowledge and Data Engineering, 18(1):1-16, January 2007.

[EJX01] D.W. Embley, D. Jackman, and L. Xu. Multifaceted exploitation of metadata for attribute match discovery in information integration. In Proceedings of the International Workshop on Information Integration on the Web (WIIW'01), pages 110-117, Rio de Janeiro, Brazil, April 2001.

[EJX02] D.W. Embley, D. Jackman, and L. Xu. Attribute match discovery in information integration: Exploiting multiple facets of metadata. Journal of the Brazilian Computing Society, 8(2):32-43, November 2002.

[EKW92] D.W. Embley, B.D. Kurtz, and S.N. Woodfield. Object-oriented Systems Analysis: A Model-Driven Approach. Prentice Hall, Englewood Cliffs, New Jersey, 1992.

$\left[\mathrm{ELL}^{+} 08\right]$ D.W. Embley, S.W. Liddle, D. Lonsdale, G. Nagy, Y. Tijerino, R. Clawson, J. Crabtree, Y. Ding, P. Jha, Z. Lian, S. Lynn, R.K. Padmanabhan, J. Peters, C. Tao, R. Watts, C. Woodbury, and A. Zitzelberger. A conceptual-modelbased computational alembic for a web of knowledge. In Proceedings of the 27th International Conference on Conceptual Modeling (ER08), pages 532533, Barcelona, Spain, October 2008.

$\left[\mathrm{ELL}^{+} 09\right]$ D.W. Embley, S.W. Liddle, D.W. Lonsdale, A. Stewart, and C. Tao. KBB: A knowledge-bundle builder for research studies. In Proceedings of 2nd International Workshop on Active Conceptual Modeling of Learning (ACM-L 2009), Gramado, Brazil, November 2009. (to appear).

[Emb80] D.W. Embley. Programming with data frames for everyday data items. In Proceedings of the 1980 National Computer Conference, pages 301-305, Anaheim, California, May 1980.

[ES07] J. Euzenat and P. Shvaiko. Ontology Matching. Springer-Verlag, Heidelberg, Germany, 2007.

[ETL02] D.W. Embley, C. Tao, and S.W. Liddle. Automatically extracting ontologically specified data from HTML tables with unknown structure. In Proceedings of 
the 21st International Conference on Conceptual Modeling (ER2002), pages 322-327, Tampere, Finland, October 2002.

[EXD04] D.W. Embley, L. Xu, and Y. Ding. Automatic direct and indirect schema mapping: Experiences and lessons learned. SIGMOD Record, 33(4):14-19, December 2004.

[Fam] Family search. http://familysearch.org.

[FM09] J.R. Finkel and C.D. Manning. Nested named entity recognition. In Proceedings of the 2009 Conference on Empirical Methods in Natural Language Processing, pages 141-150, Singapore, August 2009.

[FNS07] S.M. Falconer, N.F. Noy, and M.-A. Storey. Ontology mapping - a user survey. In Proceedings of the Second International Workshop on Ontology Mapping (OM-2007), pages 49-60, Bexco, Busan, Korea, November 2007.

$\left[\mathrm{GBH}^{+} 07\right]$ W. Gatterbauer, P. Bohunsky, M. Herzog, B. Krüpl, and B. Pollak. Towards domain-independent information extraction from web tables. In Proceedings of the Sixteenth International World Wide Web Conference (WWW2007), pages 71-80, Banff, Alberta, Canada, May 2007.

[GGTB08] C. Grover, S. Givon, R. Tobin, and J. Ball. Named entity recognition for digitised historical texts. In Proceedings of the 6th International Conference on Language Resources and Evaluation (LREC 2008), Marrakech, Morocco, May 2008.

[Gru93] T.R. Gruber. A translation approach to portable ontology specifications. Knowledge Acquisition, 5(2):199-220, 1993.

[Gua98] N. Guarino. Formal ontologies and information systems. In N. Guarino, editor, Proceedings of the First International Conference on Formal Ontology in Information Systems (FOIS98), pages 3-15, Trento, Italy, June 1998.

$\left[\mathrm{HLF}^{+} 08\right]$ L. Hunter, Z. Lu, J. Firby, W.A. Baumgartner Jr., H.L. Johnson, P.V. Ogren, and K.B. Cohen. OpenDMAP: An open source, ontology-driven, concept analysis engine, with applications to capturing knowledge regarding protein transport, protein interactions and cell-type-specific gene expression. $B M C$ Bioinformatics, 9(8), 2008.

[HRO06] A. Halevy, A. Rajaraman, and J. Ordille. Data integration: The teenage years. In Proceedings of the 32nd International Conference on Very Large Data Bases (VLDB'06), pages 9-16, Seoul, Korea, September 2006.

[JN08] P. Jha and G. Nagy. Wang notation tool: Layout independent representation of tables. In Proceedings of the 19th International Conference on Pattern Recognition (ICPR08), Tampa, Florida, December 2008.

$\left[\mathrm{JNS}^{+}{ }^{09} \quad\right.$ R.C. Jandhyala, G. Nagy, S. Seth, W. Silversmith, M. Krishnamoorthy, and R. Padmanabhan. From tessellations to table interpretation. In Lecture Notes in Artificial Intelligence, volume 5625, pages 422-437, Berlin, 2009. SpringerVerlag.

[LE09] S. Lynn and D.W. Embley. Semantically conceptualizing and annotating tables. In Proceedings of the Third Asian Semantic Web Conference, pages 345-359, Bangkok, Thailand, February 2009.

[Lia08] Z. Lian. A tool to support ontology creation based on incremental miniontology merging. Master's thesis, Department of Computer Science, Brigham Young University, Provo, Utah, March 2008.

[PJK ${ }^{+}$09] R. Padmanabhan, R.C. Jandhyala, M. Krishnamoorthy, G. Nagy, S. Seth, and W. Silversmith. Interactive conversion of large web tables. In Proceedings of Eighth International Workshop on Graphics Recognition (GREC 2009), pages 32-43, La Rochelle, France, July 2009.

[PlaBC] Plato. Theaetetus. BiblioBazaar, LLC, Charleston, South Carolina, about 360BC. (translated by Benjamin Jowett).

$\left[\mathrm{PSC}^{+} 07\right]$ A. Pivk, Y. Sure, P. Cimiano, M. Gams, V. Rajkovič, and R. Studer. Transforming arbitrary tables into logical form with TARTAR. Data $\& 3$ Knowledge Engineering, 60:567-595, 2007. 
[RB01] E. Rahm and P.A. Bernstein. A survey of approaches to automatic schema matching. The VLDB Journal, 10:334-350, 2001.

[RFRF08] D. Roussinov, W. Fan, and J. Robles-Flores. Beyond keywords: Automated question answering on the web. Communications of the ACM, 51(9):60-65, September 2008.

[RK06] F. Rahman and B. Klein. Special issue on detection and understanding of tables and forms for document processing applications. International Journal of Document Analysis, 8(2):65, 2006.

[Ros05] R. Rosati. On the decidability and complexity of integrating ontologies and rules. Journal of Web Semantics, 3(1):61-73, 2005.

[Sar08] S. Sarawagi. Information extraction. Foundations and Trends in Databases, 1(3):261-377, 2008.

[SDH08] A.D. Sarma, X. Dong, and A. Halevy. Bootstrapping pay-as-you-go data integration systems. In Proceedings of SIGMOD'08, pages 861-874, Vancouver, British Columbia, Canada, June 2008.

$\left[\mathrm{SDM}^{+} 08\right]$ W. Shen, P. DeRose, R. McCann, A. Doan, and R. Ramakrishnan. Toward best-effort information extraction. In Proceedings of the ACM SIGMOD International Conference on Management of Data, pages 1031-1042, Vancouver, British Columbia, Canada, June 2008.

[SHBL06] N. Shadbolt, W. Hall, and T. Berners-Lee. The semantic web revisited. IEEE Intelligent Systems, 21(3):96-101, May/June 2006.

[Smi03] B. Smith. Ontology. In L. Floridi, editor, Blackwell Guide to the Philosophy of Computing and Information, pages 155-166. Oxford: Blackwell, 2003.

[Tao08] C. Tao. Ontology Generation, Information Harvesting and Semantic Annotation for Machine-Generated Web Pages. PhD dissertation, Brigham Young University, Department of Computer Science, December 2008.

[TE09] C. Tao and D.W. Embley. Automatic hidden-web table interpretation, conceptualization, and semantic annotation. Data 8 Knowledge Engineering, 68(7):683-703, July 2009 .

$\left[\mathrm{TEL}^{+} 05\right] \quad$ Y.A. Tijerino, D.W. Embley, D.W. Lonsdale, Y. Ding, and G. Nagy. Toward ontology generation from tables. World Wide Web: Internet and Web Information Systems, 8(3):261-285, September 2005.

[TEL09] C. Tao, D.W. Embley, and S.W. Liddle. FOCIH: Form-based ontology creation and information harvesting. In Proceedings of the 28th International Conference on Conceptual Modeling (ER2009), pages 346-359, Gramado, Brazil, November 2009.

[Vic06] M. Vickers. Ontology-based free-form query processing for the semantic web. Master's thesis, Brigham Young University, Provo, Utah, June 2006.

[W3C] W3C (World Wide Web Consortium) Semantic Web Activity Page. http://www.w3.org/2001/sw/.

[Wan96] X. Wang. Tabular Abstraction, Editing, and Formatting. PhD thesis, University of Waterloo, 1996.

[WCW ${ }^{+}$09] J. Wang, C. Chen, C. Wang, J. Pei, J. Bu, Z. Guan, and W.V. Zhang. Can we learn a template-independent wrapper for news article extraction from a single training site? In Proceedings of the 15th ACM SIGKDD International Conference on Knowledge Discovery and Data Mining, pages 1345-1354, Paris, France, June/July 2009.

[WKRS09] G. Weikum, G. Kasneci, M. Ramanath, and F. Suchanek. Database and information-retrieval methods for knowledge discovery. Communications of the ACM, 52(4):56-64, April 2009.

[XE06] L. Xu and D.W. Embley. A composite approach to automating direct and indirect schema mappings. Information Systems, 31(8):697-732, December 2006. 
[ZBC04] R. Zanibbi, D. Blostein, and J.R. Cordy. A survey of table recognition: Models, observations, transformations, and inferences. International Journal of Document Analysis and Recognition, 7(1):1-16, 2004. 